\title{
Velocity monitoring of $\gamma$ Cas stars reveals their binarity status ${ }^{\star}$
}

\author{
Yaël Nazé ${ }^{\dagger}$, Gregor Rauw ${ }^{1}$, Stefan Czesla ${ }^{2}$, Myron A. Smith ${ }^{3}$, and Jan Robrade ${ }^{2}$ \\ ${ }^{1}$ Groupe d'Astrophysique des Hautes Energies, STAR, Université de Liège, Quartier Agora (B5c, Institut d'Astrophysique et de Géophysique), \\ Allée du 6 Août 19c, B-4000 Sart Tilman, Liège, Belgium \\ ${ }^{2}$ Hamburger Sternwarte, Universität Hamburg, Gojenbergsweg 112, 21029 Hamburg, Germany \\ ${ }^{3}$ NSF OIR Lab, 950 N Cherry Ave, Tucson, AZ 85721, USA
}

19 November 2021

\begin{abstract}
The binary status of $\gamma$ Cas stars has been discussed while theoretically examining the origin of their peculiar X-ray emission. However, except in two cases, no systematic radial velocity monitoring of these stars had been undertaken yet to clarify their status. We now fill this gap using TIGRE, CARMENES, and UVES high-resolution spectroscopy. Velocities were determined for 16 stars, revealing shifts and/or changes in line profiles. The orbit of six new binaries could be determined: the long periods $(80-120 \mathrm{~d})$ and small velocity amplitudes $\left(5-7 \mathrm{~km} \mathrm{~s}^{-1}\right)$ suggest low mass companions $\left(0.6-1 \mathrm{M}_{\odot}\right)$. The properties of the known $\gamma$ Cas binaries appear similar to those of other Be systems, with no clear-cut separation between them. One of the new systems is a candidate for a rare case of quadruple system involving a Be star. Five additional $\gamma$ Cas stars display velocity variations compatible with the presence of companions, but no orbital solution could yet be formally established for them hence they only receive the status of "binary candidate".
\end{abstract}

Key words: stars: early-type - stars: massive - stars: emission-line,Be - binaries: general - stars: variables: general

\section{INTRODUCTION}

The Be star category was first described nearly 150 years ago with the discovery of emission lines in $\gamma$ Cas, the central star of Cassiopeia's "W" (Secchi 1866). These lines are now thought to arise in a viscuous decretion disk (for a review, see Rivinius et al. 2013). However, the prototype star, $\gamma \mathrm{Cas}$, was found to be quite atypical amongst its defining class. Indeed, it emits very bright and extremely hard X-rays with a luminosity intermediate between that of "normal" massive stars and that of high-mass X-ray binaries (for a review, see Smith et al. 2016). Over the last two decades, it was discovered that $\gamma$ Cas is not a lone bird, hence the definition of a " $\gamma$ Cas" category. Currently, $25 \mathrm{Be}$ stars are known to display similar X-ray properties (see Nazé \& Motch 2018 and Nazé et al. 2020a): such $\gamma$ Cas objects thus represent a non-negligible fraction of the classical Be population.

The mechanism behind the peculiar X-ray emission of $\gamma$ Cas analogs is still debated, with a handful scenarios having been proposed. The first one involves accretion onto a compact companion (white dwarf, WD, Murakami et al. 1986; neutron star, NS, in a propeller regime, Postnov et al. 2017). A second one imagines the collision between the stellar wind of a hot, non-degenerate, companion and the Be disk (Langer et al. 2020). A third one considers stardisk interactions involving localized magnetic fields, generated by disk instabilities (Smith et al. 1998; Smith \& Robinson 1999), and stellar fields arising from a subsurface equatorial convective zone (Cantiello et al. 2009; Motch et al. 2015). A crucial difference be-

\footnotetext{
$\star$ Based on spectra obtained with TIGRE-HEROS, VLT-UVES, and CAHACARMENES

† F.R.S.-FNRS Senior Research Associate, email: ynaze@uliege.be
}

tween these scenarios is the presence (and nature) of companions. If either the accretion scenario or the wind collision idea is correct, all $\gamma$ Cas stars must have a companion. In the first case, this companion would be a compact object with a mass typical of either a white dwarf or a neutron star. In the second case, the companion would be a stripped Helium star whose mass would span the range expected for such objects (typically a few $M_{\odot}$, the exact value being linked to the Be star mass, see Shao \& Li 2021). For the last scenario, companions are possible, but their presence is certainly not a requirement since $\mathrm{X}$-ray production is not linked to the companion. Consequently, assessing the systematic presence of close companions to $\gamma$ Cas stars constitutes a crucial step towards constraining the nature of their peculiar X-ray emission.

Furthermore, the binarity status is of particular interest in the context of evolutionary models (Shao \& Li 2014, 2021). Indeed, in the first scenario, $\gamma$ Cas stars could fill the theoretically-predicted population of Be+WD systems (Raguzova 2001), which should largely outnumber Be+NS X-ray binaries although only a handful of cases are known. Alternatively, the detection of neutron star companions would lead to an increase in the number of NS+OB systems, also impacting on evolutionary theories. Finally, the presence of nondegenerate companions would lead to constraints on the origin of Be stars themselves, in addition to information on stellar evolution. Indeed, the Helium stars have lost their envelope after a mass-transfer episode and, in such an event, angular momentum is also transfered: the associated spin-up would then explain the fast rotation of Be stars (Shao \& Li 2014).

Amongst the 25 known $\gamma$ Cas stars, two are known binaries: $\gamma$ Cas itself (e.g. Nemravová et al. 2012; Smith et al. 2012) and $\pi$ Aqr (Bjorkman et al. 2002; Nazé et al. 2019a). The velocity variations of a third object, HD 45314, were examined by Rauw et al. (2018) who 
found no evidence for periodic velocity variations with amplitude $>10 \mathrm{~km} \mathrm{~s}^{-1}$. For the other $\gamma$ Cas stars, systematic monitorings have not yet been done. Thanks to dedicated pilot programmes, we begin this endeavour using TIGRE and CARMENES for seven northern targets and UVES for nine southern targets (see further details below). The six remaining $\gamma$ Cas stars are too faint for a detailed study with current facilities. Section 2 presents the obtained observations as well as the velocity mesurement methods. Section 3 examines the results obtained, with a specific focus on the new detections. Section 4 discusses the results, while the last section summarizes our findings.

\subsection{The binarity of Be stars}

Before examining our results and their impact on the debate regarding the $\gamma$ Cas phenomenon, the more general question of multiplicity in the overall Be category should also be presented. It has been known for decades that some Be stars are binaries. Indeed, tens of them show up brightly at X-ray or gamma-ray wavelengths (Walter et al. 2015, and references therein). In such cases, the Be star is paired with a neutron star (NS) which accretes material from its companion, explaining the intense high-energy emission. Of course, this bright emission makes them detectable from afar and the sheer number of such systems may induce a false idea of great abundance. Considering volume-limited samples, such X-ray binaries actually represent only a small fraction of the Be population. Theoretical simulations by Raguzova (2001) indeed predict $70 \%$ of white dwarf (WD) companions, $20 \%$ of stripped, He-star companions, and only $10 \%$ of NS companions in Be binaries. More recently, Shao \& Li (2014) provided additional support to a low fraction of NS companions as they calculated that there should be $50 \mathrm{Be}+\mathrm{NS}$ systems for each $\mathrm{Be}+\mathrm{BH}$ case, but a thousand other Be binary systems for each Be+NS case. This thousand should be equally split between Be stars with WD companions and Be stars with stripped, He-star companions. Furthermore, binary properties may differ between these different cases. For example, both Raguzova (2001) and Shao \& Li (2014) expect a large range of eccentricities for NS companions, depending on the supernova nature and kick. In contrast, more circular systems are generally expected when mass transfer has occurred but no supernova has taken place, as for WD and He-star companions (although some simulations suggest non-zero eccentricities, see Sepinsky et al. 2009; Dosopoulou \& Kalogera 2016). Regarding orbital periods, Raguzova (2001) favor larger values for NS (100-3000 d) then for WD and Hestar companions (20-300 d) while Shao \& Li (2014) found similar ranges (20-2000 d) in all cases. Shao \& Li $(2014,2021)$ also expected a correlation between the mass of the Be star and the mass of the He-star: the stripped companion should have a mass of $0.5-3 \mathrm{M}_{\odot}$ for a Be mass of $6-15 \mathrm{M}_{\odot}$. Furthermore, for that range of the $\mathrm{Be}$ mass, the period distribution appeared rather flat.

The difficulty of detecting these other pairs may strongly bias the observed incidence of such companions, however. For example, the expected large population of Be stars with WD companions has not been found yet. Only a few cases were reported, e.g. through the detection of Be stars associated to supersoft X-ray sources (e.g. Cracco et al. 2018; Kennea et al. 2021). In parallel, the UV radiation of hot, stripped He-stars allowed to detect such companions, but only for a few Be stars as the detection is here also very challenging (Wang et al. 2018, 2021). Finally, while orbits could be established for some Be binaries (see Sect. 4 below), the companion rarely (if at all!) seems to be a main-sequence star with similar mass. For example, Bodensteiner et al. (2020) mentioned a clear lack of massive companions (with mass ratios $>0.2$, i.e. A- and B-type) to early-type Be stars, compared to what is found in other B-type stars.

Thus, the overall picture that emerges from previous research is that Be stars may be found in binary systems, often (if not always) with an evolved companion. The following question then concerns the actual fraction of Be binaries amongst the Be population. This is often debated in the context of evolutionary models and the emergence of Be signatures. Indeed, the Be stars are known to be fast rotators, and this is considered as an essential property to achieve the Be phenomenon (although probably not the sole one as some fast rotating B-stars are not of the Be type). There are two ways to obtain such a fast rotation. On the one hand, one may consider B-type stars as being born with a range of rotational velocities and only the fastest rotators turning into Be stars (with or without the need of a further spinup during main sequence, see Zorec \& Briot 1997 vs. Ekström et al. 2008). In this case, binarity brings "no extra clue on the origin of the Be phenomenon" (Baade 1992) as it constitutes an unrelated factor. On the other hand, one may consider the fast rotation as acquired after a transfer of angular momentum in an interacting binary system. A first proposal had been that Be stars correspond to systems currenly undergoing mass-transfer, with the Be disk being an accretion feature (e.g. Kriz \& Harmanec 1975). This scenario was later discarded as such a phase is short-lived and the necessary mass-losing companions (cool giants) were not detected in most Be stars. Therefore, the binary idea was restricted to post mass-transfer systems. In such a case, the donor star would then appear as a stripped star which could end up as a WD or NS. Theoretical estimates of the relative importance of one channel over the other have been done, with widely different results. For example, van Bever \& Vanbeveren (1997) favored little contribution (maximum $20 \%$ of all Be stars) of the binary channel, whereas Pols et al. (1991) or Shao \& Li (2014) considered it much more dominant (40-60\% of Be stars for the former, $\sim 100 \%$ for the latter).

To estimate the overall binarity fraction of Be stars, specific observational studies have also been undertaken. Boubert \& Evans (2018) estimated the fraction of runaways amongst Be stars and found that it agrees with expectations for post mass-transfer systems, suggesting the binary channel to be dominant. Klement et al. (2019) examined the SED of a sample of Be stars. In these stars, the SED combines the usual stellar photospheric emission with the emission from the cooler disk. Klement et al. (2019) found SED turndowns at radio wavelengths for all stars with sufficient wavelength coverage. They interpreted that result as evidence for disk truncation by companions, suggesting that the vast majority of Be stars possess companions and are born through the mass-transfer channel. Finally, Hastings et al. (2021) could reconcile the fraction of Be stars observed in clusters with predictions of binary models. However, they noted that, "if all Be stars are binary interaction products, somewhat extreme assumptions must be realised" for the match to occur. In particular, all stars are binaries in their model and, whatever the stellar and orbital properties, each system undergoes a fully non-conservative mass-transfer immediately at the end of the main sequence, which necessarily leads to the formation of a Be star.

\section{OBSERVATIONS AND DATA REDUCTION}

The list of observed stars and their basic properties is provided in Appendix A (Table A1). 


\subsection{CARMENES}

Four northern stars (V782 Cas=HD 12882, V810 Cas=HD 220058 , V2156 Cyg, and SAO 49725) were observed with the CARMENES (Calar Alto high-Resolution search for M dwarfs with Exoearths with near-IR and optical Echelle Spectrographs, Quirrenbach et al. 2018) instrument. All data were obtained in service mode via the OPTICON common time allocation process (programs ID 19B/001+21B/017) at a rate of about one observation every two weeks in AugustNovember 2019 and July-October 2021. Installed on the $3.5 \mathrm{~m}$ telescope of the Centro Astronómico Hispano Alemán at Calar Alto Observatory (Spain), CARMENES records the stellar spectra thanks to two separate echelle spectrographs, one for red wavelengths (5200-9600 $\AA, R=94600)$ and one for the near-IR $(0.96-1.71 \mu \mathrm{m}$, $R=80400)$. The spectra were reduced using the CARMENES pipeline (Caballero et al. 2016). The individual spectra were taken with exposure times between 1 and 15 min depending on star brightness and weather conditions. If obtained the same night, they were combined to improve signal-to-noise ratios. The final spectra (about ten for each star) display typical signal-to-noise ratios of 150-200. Within IRAF, a further correction was made for eliminating telluric lines around $\mathrm{H} \alpha$ and $\mathrm{He}_{\mathrm{i}} \lambda 5876 \AA$ in the optical (template of Hinkle et al. 2000), as well as around $1 \mu \mathrm{m}$ and $1.28 \mu \mathrm{m}$ in the near-IR (template as in Nazé et al. 2019b). Sky emission lines near $1.56 \mu \mathrm{m}$ were also eliminated, using the data from the sky fiber. As a last step, the spectra were normalized over limited wavelength windows using splines of low order.

\subsection{TIGRE}

Four bright northern/equatorial stars (V782 Cas=HD 12882, V558 Lyr=HD 183362, HD 44458=FR CMa, and HD 45995) were observed between November 2018 and October 2021 with the fully robotic $1.2 \mathrm{~m}$ TIGRE telescope located in central Mexico (Schmitt et al. 2014). A typical bimonthly cadence was used over the visibility period, weather permitting: in total, we collected 33 spectra of V782 Cas, 16 spectra of V558 Lyr, 20 spectra of HD 44458, and 19 spectra of HD 45995. As a complement to the CARMENES observations, three spectra of V810 Cas and V2156 Cyg, plus one of SAO 49725 were also collected.

TIGRE is equipped with the HEROS echelle spectrograph (3800$5700 \AA+5800-8800 \AA, R \sim 20000)$. The data reduction was performed with the dedicated TIGRE/HEROS reduction pipeline (Mittag et al. 2011). Absorption by telluric lines was corrected within IRAF using the template of Hinkle et al. (2000) around He I $\lambda 5876 \AA$ and $\mathrm{H} \alpha$. As a last step, the high-resolution spectra were normalized over limited wavelength windows using splines of low order. Typical exposure times were between 5 and $30 \mathrm{~min}$, depending on star brightness and weather conditions; typical signal-to-noise ratios (after box smoothing over 7 pixels) were about 200. Five spectra of V782 Cas showing very low signal-to-noise ratios were discarded, as well as another one of the same star showing a deviating velocity for the interstellar Ca II 3934 Å line.

\subsection{UVES}

Nine southern stars (HD 90563, HD 110432=BZ Cru, HD 119682, V767 Cen=HD 120991, CQ Cir=HD 130437, HD 157832=V750 Ara, HD 161103=V3892 Sgr, V771 Sgr=HD 162718, and HD 316568) were observed with the Ultraviolet and Visual Echelle Spectrograph (UVES, Dekker et al. 2000), installed on the second Unit Telescope (UT2) at the Cerro
Paranal ESO Observatory, for our ESO program ID 105.204D. Spectra were taken on five dates between September 2020 and March 2021, with at least 10 days between successive exposures. UVES was used in dichroic mode, allowing simultaneous access to the $3300-4560 \AA$ and $4730-6830 \AA$ regions (with a $80 \AA$ gap near $\sim 5800 \AA$ ). Exposure times ranged between 0.25 and 30 min, depending on star brightness and weather conditions, leading to typical signal-to-noise ratios of 100-150 in the blue and 200-250 in the red. The slit width was $0.4^{\prime \prime}$ in the blue and $0.3^{\prime \prime}$ in the red, leading to $R \sim 70000$ and 100000 , respectively. The data were reduced in a standard way by ESO pipeline. Telluric lines were corrected near $\mathrm{He}_{\mathrm{I}} \lambda 5876 \AA$ and $\mathrm{H} \alpha$ in the same way as for CARMENES and TIGRE spectra. Finally, the spectra were normalized over the same set of continuum windows using polynomials of low order. Spectra were taken in service mode, but were not always checked for saturation in $\mathrm{H} \alpha$ : only some saturated observations were repeated with a reduced exposure time - fortunately, the other lines are unaffected by this problem.

\subsection{TESS}

For V782 Cas, additional photometric data have been obtained by TESS since those presented in Nazé et al. (2020c) hence we reextracted the whole dataset. The $30 \mathrm{~min}$ lightcurves of both sectors (18 and 25) were derived through aperture photometry using the Python package Lightkurve ${ }^{1}$. On image cutouts of $50 \times 50$ pixels, we defined a source mask from pixels presenting fluxes well above the median flux (10 or 25 Median Absolute Deviation over it) and a background mask from pixels with fluxes below the median flux. The background contamination was then corrected using a principal component analysis with 5 components. All data points with errors larger than the mean of the errors plus three times their $1 \sigma$ dispersion were discarded. The TESS fluxes were converted into magnitudes using $\operatorname{mag}=-2.5 \times \log ($ flux $)$, the mean magnitude in each sector was subtracted, and then the data were combined into a final TESS lightcurve $^{2}$.

\subsection{Measuring velocities of Be stars}

Our aim is to detect binary motion through radial velocity measurements. However, several phenomena can lead to velocity changes, fortunately with different properties. Binary motion should affect all lines of a given star in the same way, while pulsations or disk evolution will show up differently depending on line. This allows us to disentangle these effects: only stars showing velocity shifts which are coherent from line to line will be considered as "binary candidates". If a period and a full orbital solution can be calculated, then the status becomes secure and the "candidate" is dropped.

However, measuring radial velocities $(R V s)$ in Be stars is not a simple endeavour, as their lines are broad, with few and faint photospheric lines being present while emission lines may be strong but display variable profiles due to disk build-up or dissipation events. To characterize the lines, we therefore (1) carefully selected them and (2) used several methods to constrain their $R V s$.

To get reliable $R V s$, strong lines must be studied. For CARMENES spectra, we chose lines from hydrogen ( $\mathrm{H} \alpha$, Paschen lines at 0.8598 , $0.8750,1.00$, and $1.28 \mu \mathrm{m}$, plus the Brackett line at $1.56 \mu \mathrm{m})$ as

\footnotetext{
1 https://docs.lightkurve.org/

2 Note that this new reduction of the TESS data of V782 Cas in Sector 18 produce a lightcurve fully agreeing with that reported in Nazé et al. (2020c).
} 

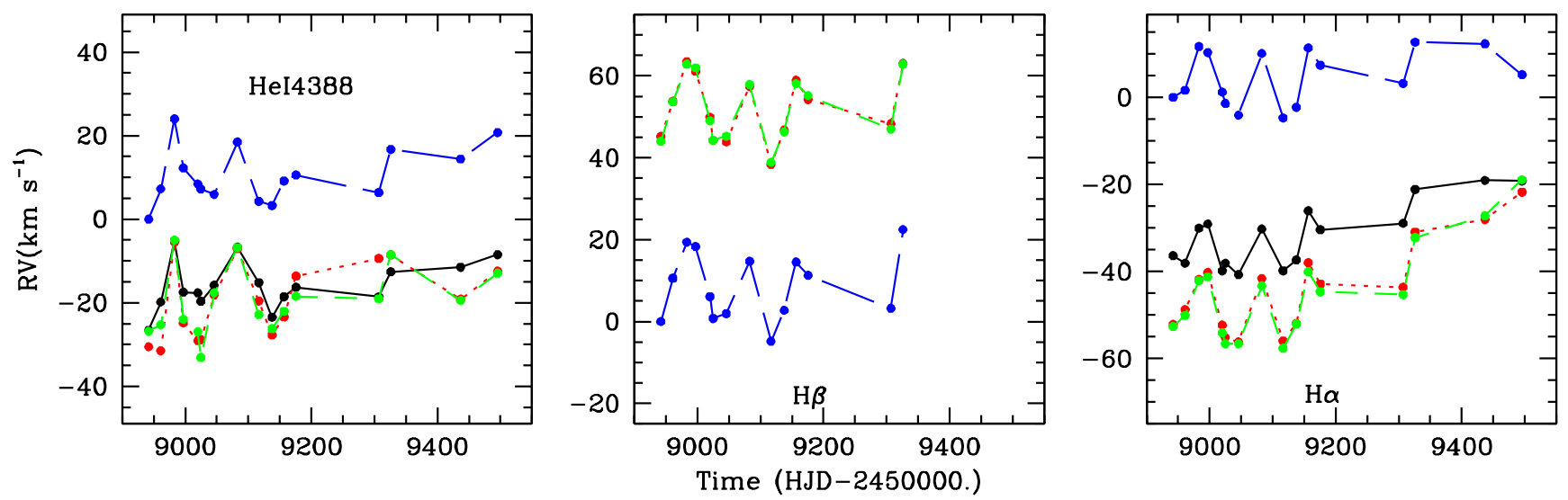

Figure 1. Radial velocities measured for V558 Lyr on He I $4388 \AA$ (left), $\mathrm{H} \beta$ (middle) and $\mathrm{H} \alpha$ (right) lines using the moment method (black points and solid line), the mirror method (red points and dotted line), the double Gaussian method (green points and dashed line), and the correlation method (blue points and long dashed line). Note that the mix of absorption and emission in $\mathrm{H} \beta$ makes the first order moments unreliable hence they are not shown.

well as from He I $5876 \AA$ and Fe II $9998 \AA$. For TIGRE and UVES data, we focused on lines from hydrogen ( $\mathrm{H} 8, \mathrm{H} \beta, \mathrm{H} \alpha)$ and helium ( $\mathrm{He}_{\mathrm{I}} 4026,4143,4388,4471,5876,6678 \AA$ ). Of those sets, H8 is the only hydrogen line with a dominant absorbing component. The line is however very broad and quite noisy, making it unreliable. Lines of He I 5876,6678 $\AA$ usually show a mix of absorption and emission, while bluer He I lines appear dominated by absorption. In addition to stellar lines, we also studied interstellar lines (Ca II $3934 \AA$, Na I $5895 \AA$, DIB at $5780 \AA$ ) to get an idea of the precision of the $R V$ measurement methods.

Four methods were used to measure the $R V s$. The first one calculates the first-order moment $M_{1}$ using $M_{1}=\sum\left(F_{i}-1\right) \times v_{i} / \sum\left(F_{i}-1\right)$ (Nazé et al. 2019a), where $v_{i}$ the velocity of the ith spectral bin and $F_{i}$ the normalized flux at the ith spectral bin. This method appears quite robust in presence of noise since it considers the whole line profile. For the same reason, however, it is also sensitive to profile changes. This may become particularly problematic for lines strongly affected by disk emission (e.g. $\mathrm{H} \alpha$ emissions) as the line core, linked to the outer part of the disk, is often quite variable. In addition, it provides erratic results if the equivalent width is small (because the denominator is then close to zero), i.e. it does not work well for lines mixing absorption and emission of similar amplitudes.

The second method is the correlation method. It compares one spectrum (taken as reference) to all others for a set of velocity shifts. The best shift is found by ways of a $\chi^{2}$ minimization and a parabolic fit to the minimum $\chi^{2}$ value and its neighbours is then made to find the final value of the relative velocity. Like the previous method, it uses the whole line profile hence may become biased if the profile strongly changes. However, it does not have any problem with mixed absorption/emission profiles.

The next two methods put emphasis on the wings of the line profile, avoiding the line core. They both require the lines to have quite large amplitudes, compared to the noise. The mirror method compares, for several velocity shifts, the blue wing to the mirrored red wing (i.e. after reversing velocities, Nemravová et al. 2012 and references therein). Only the wings between approximately $20 \%$ and $60 \%$ of the maximum amplitude were considered in this calculation, as done for $\gamma$ Cas (Nemravová et al. 2012). The least difference between the wings is searched for and a parabolic fit to the minimum $\chi^{2}$ value and its neighbours is made to get the final velocity value. While it avoids the core, this method requires the wings to be spread over a number of pixels: it is thus difficult to use on steep lines such as interstellar lines.

The double Gaussian technique correlates the line profile to the function $G(v)=\exp \left[-(v-a)^{2} / 2 \sigma^{2}\right]-\exp \left[-(v+a)^{2} / 2 \sigma^{2}\right]$ where $v$ is the velocity, $a$ the center of the positive Gaussian function $(-a$ is used for the negative one), and $\sigma$ the standard deviation of both Gaussian functions (Smith et al. 2012, based on Shafter et al. 1986). The $R V$ corresponds to the shift at which the correlation reaches zero. In each case, we adjust parameter $a$ to put maximum weight where the line reaches half maximum (i.e. it is a bisector value at half amplitude). The width $\sigma$ was chosen to be $10 \mathrm{~km} \mathrm{~s}^{-1}$ (for CARMENES and UVES) or $15 \mathrm{~km} \mathrm{~s}^{-1}$ (for TIGRE) for stellar lines and $5 \mathrm{~km} \mathrm{~s}^{-1}$ for the narrower interstellar lines.

Because these four methods are sensitive to different aspects of the line profile and because the line shape (e.g. asymmetric profiles) may skew the results of one method but not another, the absolute velocity values found by these four methods may not agree. However, for simple cases (e.g. no strong changes of line core), their relative variations should agree. Note that we determined width and skewness of each line in each star, in order to check for the presence of large line profile changes. Except for the last spectra of V558 Lyr, the northern targets displayed relatively stable profiles, while this was much less the case for southern stars. Nevertheless, aside from interstellar lines, the $\mathrm{H} \alpha$ line appears as the most stable line in the vast majority of cases.

\section{RESULTS}

Whatever the method, the velocities derived for the narrow interstellar lines (Ca II and/or Na I) yield dispersions of $\sim 1 \mathrm{~km} \mathrm{~s}^{-1}$ for TIGRE spectra, $\sim 0.1 \mathrm{~km} \mathrm{~s}^{-1}$ for CARMENES spectra, and $\sim 0.15 \mathrm{~km} \mathrm{~s}^{-1}$ for UVES spectra. This corresponds well to the expected resolution and wavelength calibration limits. For the broader and very shallow DIB recorded on CARMENES spectra, the dispersion however rises to $\sim 1 \mathrm{~km} \mathrm{~s}^{-1}$. Although the stellar lines are less shallow, the actual uncertainty on their velocities may thus be somewhat larger than for narrow lines. 
Four stars were repeatedly observed with TIGRE, thereby allowing a finer study. Indeed, whenever recurring variations are found, an orbital solution could be derived since there were enough spectra. The specific results on these four stars are thus discussed in separate subsections (Sect. 3.1-4). The CARMENES+TIGRE observations of three additional targets are fewer in number but provided first hints towards orbital solutions, presented in Sect. 3.5. Finally, the UVES datasets of other $\gamma$ Cas stars only allow for a preliminary assessment, hence they are discussed in the last subsection (Sect. 3.6). For all stars, the Appendix provides the full list of measured velocities (Tables A2 to A18). Note that all orbital solutions derived below use simple sinusoids, i.e. consider zero eccentricity: this comes from (1) the small eccentricity revealed by the phase-folded velocities and (2) the quality of the data (limited number of observations, velocity uncertainties) especially in view of the small orbital motions detected.

\subsection{V558 Lyr}

Up to now, no companion was reported in the literature (Abt \& Cardona 1984; Becker et al. 2015, although only 3 spectra taken the same day were studied in the latter case). However, our radial velocities of V558 Lyr clearly display recurrent variations, whatever the method used to measure them (Fig. 1). These variations are best seen for the $\mathrm{H} \beta$ and $\mathrm{H} \alpha$ lines. The very broad $\mathrm{H} 8$ line shows variations of an overall similar character but much noisier, rendering its results unusable. For the He I lines, which are in absorption, the same wiggle is seen but superimposed on an increasing trend for all lines but $\mathrm{He}_{\mathrm{I}} 4388 \AA$. The fact that the motion truly seems to affect the whole spectrum indicates without ambiguity the signature of binary motion. No significant trace of lines from the companion are seen in our spectra (e.g. Calcium triplet in the near-IR), unfortunately, so that V558 Lyr can only be classified as SB1 for the moment.

In the most recent spectra, line profile changes are detected. For example, the $\mathrm{H} \beta$ line showed a double-peaked line profile with a strongly dominating red peak until April 2021. Afterwards, in the last two spectra, the peaks become more equal, with the blue peak finally becoming the strongest one. Similar blue/red variations are detected in other lines. To avoid any confusion in the nature of the velocity shifts, we discarded the last two spectra from further consideration in the orbital derivation.

We performed a period search on $\mathrm{H} \alpha$ velocities (derived with the mirror method), using several methods (modified Fourier periodogram Heck et al. 1985; Gosset et al. 2001; Zechmeister \& Kürster 2009, AOV Schwarzenberg-Czerny 1989, conditional entropy Cincotta et al. 1999; Cincotta 1999; Graham et al. 2013). A peak is clearly detected at $83.3 \pm 1.8 \mathrm{~d}$ in all methods (e.g. Fig. 2). It appears at 2.6 times the mean level of the modified Fourier periodogram, but the fact that the modulation is seen on several lines leaves little doubt on the binary nature. Only with more data (there are only 14 points after all!) can the peak better stand out and a more precise period value be found. A $\chi^{2}$ fit yields a best-fit sinusoid with parameters: $T_{0}=2459056.2 \pm 1.4$ (conjunction with the Be primary in front, Fig. 3) and $K=8.2 \pm 1.1 \mathrm{~km} \mathrm{~s}^{-1}$. The $\mathrm{H} \alpha$ velocity dispersion passes from an initial $7 \mathrm{~km} \mathrm{~s}^{-1}$ to only $3 \mathrm{~km} \mathrm{~s}^{-1}$ once that best-fit sinusoid is taken out.

The best-fit parameters yield a mass function of $0.0048 \pm 0.0019 M_{\odot}$. Considering the Be star (spectral type $\mathrm{B} 3 \mathrm{~V}$ ) to have a mass of $8 M_{\odot}$ (Vieira et al. 2017) and the system's inclination to be $60-90^{\circ}$, the companion should then have a mass of $0.7-0.8 \pm 0.1 M_{\odot}$. The values of period and velocity amplitude derived for the Be star in V558 Lyr are similar to those found for

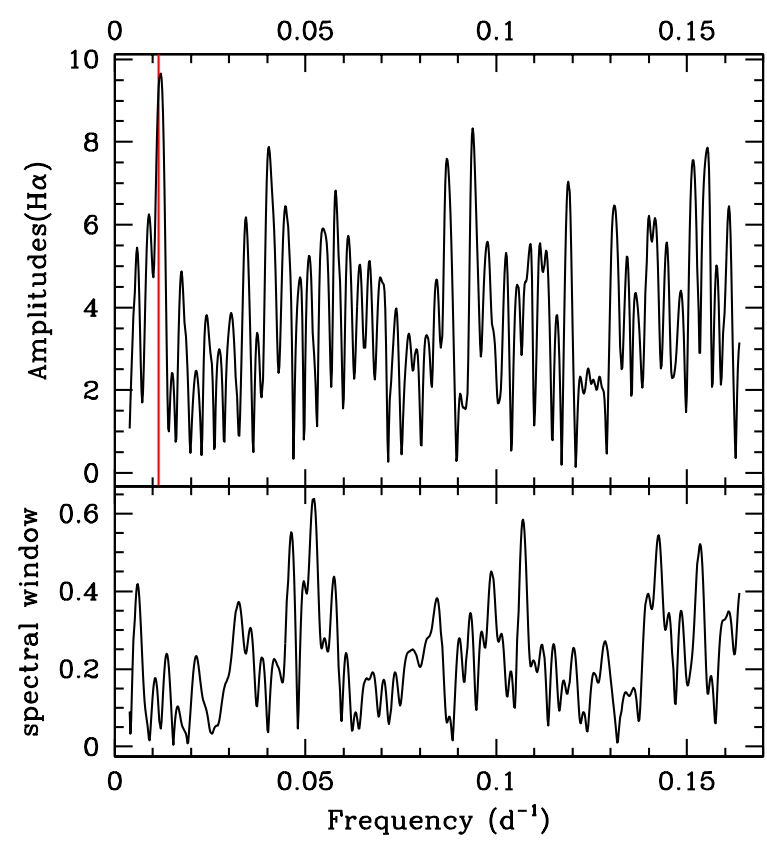

Figure 2. Periodogram derived for $\mathrm{H} \alpha$ velocities of V558 Lyr measured with the mirror method by the modified Fourier algorithm, along with its spectral window. A vertical line indicates the proposed orbital period.

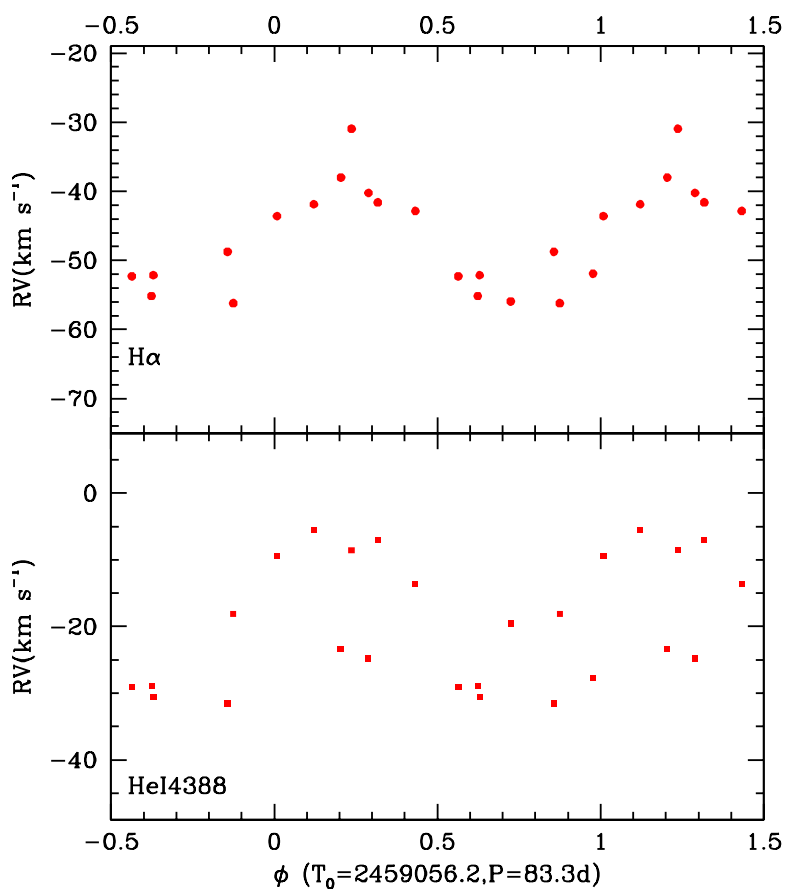

Figure 3. Velocities measured for V558 Lyr with the mirror method for He I $4388 \AA$ (bottom) and $\mathrm{H} \alpha$ (top) folded with the best-fit ephemeris. 

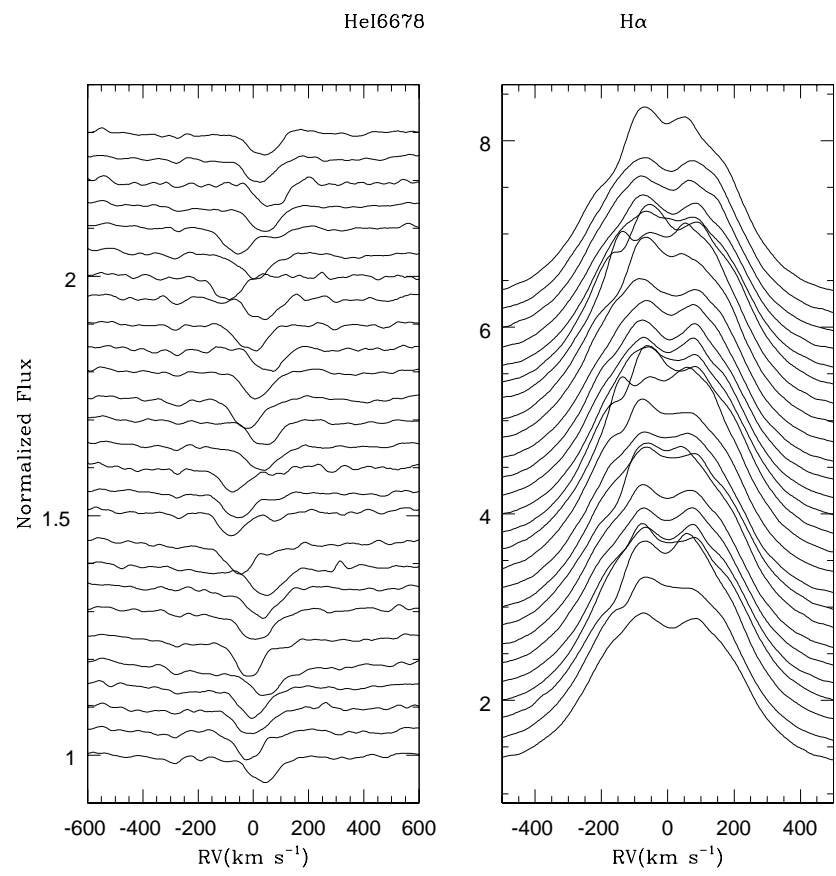

Figure 4. Evolution with time of the He I $6678 \AA$ (left) and $\mathrm{H} \alpha$ (right) line profiles observed for V782 Cas with TIGRE. Time is running downwards.

HR 2142 (Peters et al. 2016) although the type of Be primary of the latter system (B1.5IV-V) appears earlier than for the Be star in V558 Lyr. It may be noted in this context that no hint of a "hot" companion (i.e. stripped Helium star) was found for V558 Lyr by Wang et al. (2018). However, this was also the case for HR 2142: its companion is too faint hence its presence is not easily detected. Therefore, the possibility of a hot companion to V558 Lyr cannot be dismissed on that basis.

\subsection{V782 Cas}

\subsubsection{Discovery of a double periodicity}

On both CARMENES and TIGRE spectra, a very distinct and rather narrow absorption feature is clearly seen in the He I 5876,6678 lines of V782 Cas. That component covers $\sim 220 \mathrm{~km} \mathrm{~s}^{-1}$ and has $F W H M \sim 130 \mathrm{~km} \mathrm{~s}^{-1}$. It presents substantial shifts from one observation to the next (left panel of Fig. 4). The He I lines at lower wavelengths appear broader (covering $\sim 700 \mathrm{~km} \mathrm{~s}^{-1}$ ), with the narrow component buried in the overall profile. The $\mathrm{H} \alpha$ profile also presents variations, but of a different phenomenology (right panel of Fig. 4).

The difference in behaviour is contrasted in Figs. 5 and 6: the He I velocities vary by a large amount (up to $150 \mathrm{~km} \mathrm{~s}^{-1}$, Fig. 5) on short timescales whereas the $\mathrm{H} \alpha$ velocities rather display a smooth, shallower (15 $\mathrm{km} \mathrm{s}^{-1}$ amplitude), and longer-term modulation (Fig. 6). Performing period searches on the set of TIGRE velocities (because they are more numerous) clearly confirm the difference (Fig. 7), with periods of $2.5132 \pm 0.0006 \mathrm{~d}$ found for $\mathrm{He}_{\mathrm{I}}$ lines and $122.0 \pm 1.5 \mathrm{~d}$ found for $\mathrm{H} \alpha$. The peaks reach amplitudes of $\sim 4$ times the mean level of their respective periodograms, and it is important to note that no trace of the $2.5 \mathrm{~d}$ signal is seen in $\mathrm{H} \alpha$ periodogram and of the $122 \mathrm{~d}$ signal in He I periodogram (Fig. 7).

The shorter period is reminiscent of the photometric periodicity reported by Labadie-Bartz et al. (2017) and Nazé et al. (2020c). In
Table 1. Estimated properties of the components in V782 Cas. Note that the value of $57 \%$ corresponds to the combined contribution of component A (i.e. $\mathrm{Aa}+\mathrm{Ab}$ ) to the total light.

\begin{tabular}{cccc}
\hline Name & sp. type & $\begin{array}{c}M \\
\left(\mathrm{M}_{\odot}\right)\end{array}$ & $\begin{array}{c}\text { light } \\
\text { fraction }\end{array}$ \\
\hline $\mathrm{Aa}$ & $\mathrm{B} 2.5 \mathrm{III}$ & 9 & $57 \%$ \\
$\mathrm{Ab}$ & & 0.65 & \\
$\mathrm{Ba}$ & $\mathrm{B} 2$ & 9 & $35 \%$ \\
$\mathrm{Bb}$ & $\mathrm{B} 5$ & 6 & $8 \%$ \\
\hline
\end{tabular}

the latter reference, those photometric changes were interpreted as signatures from eclipses in a system unrelated to the Be star. This is reinforced by the fact that the $R U W E$ (Renormalised Unit Weight Error) parameter in Gaia-DR3 catalog is 1.87 , a large value being generally indicative of a very close optical companion ${ }^{3}$. The recorded spectra would then be a superposition of the signatures of the $\mathrm{Be}$ system (a component which we call A) and of the lines from a binary (a component which we call B), see Table 1. This helps explaining the weird line behaviour mentioned above. Most probably, the Be contribution to $\mathrm{He}_{\mathrm{I}} 5876,6678 \AA$ is filled-in by emission, as often encountered in Be stars. With such an absorption/emission balance, the Be contribution becomes indistinguishable from the continuum, leaving only the contribution from the primary of the eclipsing system detectable. On the other hand, no (or little) emission contaminates the other $\mathrm{He}$ I lines, allowing both the broad Be absorption and the narrower component of the eclipsing system to be detected.

The blue $\mathrm{He}$ I lines therefore contain the contributions from two stars and the velocity measurement methods have different sensitivities to this mixing. The correlation method provides results clearly dominated by the narrow component, whereas the moment method rather yields a sort of average. Indeed, the velocity amplitude derived from moments appears to decrease when the Be absorption becomes more dominant (i.e. for blue He I lines). Finally, wing methods have difficulties to provide sensible results, especially because of the rather large noise of blue $\mathrm{He}$ I lines.

\subsubsection{V782 Cas B, the short-period binary}

We used the velocities of the He r lines determined by the correlation method to determine a preliminary orbital solution. Averaging on all $\mathrm{He}_{\mathrm{I}}$ lines, the best-fit sinusoid has an amplitude of $64 \pm 10 \mathrm{~km} \mathrm{~s}^{-1}$ with $T_{0}=2458519.151 \pm 0.013$ (conjunction with the primary star in front). The velocity dispersion passes from an initial $40 \mathrm{~km} \mathrm{~s}^{-1}$ to only $7 \mathrm{~km} \mathrm{~s}^{-1}$ once that best-fit sinusoid is taken out. It may further be noted that forcing the fitting to $\mathrm{H} \alpha$ velocities by a sinusoid with a period fixed to $2.5 \mathrm{~d}$ yields no change in velocity dispersion, in line with the absence of a peak at that frequency in the periodogram (see also right panel of Fig. 5). Since He I lines are detected for the primary of the short-period binary despite the contribution of the bright $\mathrm{Be}$ star and since the $\mathrm{He}_{\mathrm{I}}$ lines reach their maximum strength at spectral type $\mathrm{B} 2$, we conclude that the brightest component of this binary is likely near spectral type B2.

To further study this short-period binary, we have applied our spectral disentangling code (e.g. Rosu et al. 2020) based on the shift-andadd method of González \& Levato (2006). In most spectral regions,

\footnotetext{
3 https://gea.esac.esa.int/archive/documentation/GDR2/

Gaia_archive/chap_datamodel/sec_dm_main_tables/ssec_dm_ruwe.html
} 

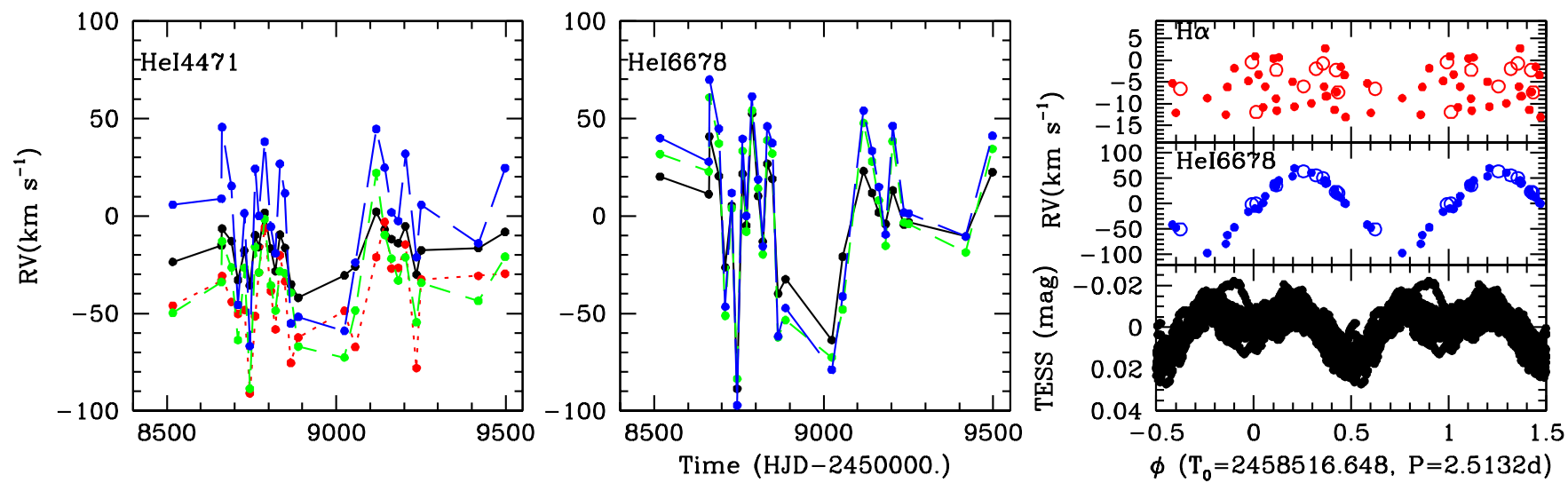

Figure 5. Left and Middle: TIGRE radial velocities measured for V782 Cas on He I 4471,6678 A (left and middle) using the moment method (black points and solid line), the mirror method (red points and dotted line), the double Gaussian method (green points and dashed line), and the correlation method (blue points and long dashed line). Note that the mirror method sometimes fails for the He I $6678 \AA$ because of the narrowness of the line, hence its associated velocities are not shown. Right: TESS photometry, He I velocities (derived by the correlation method) and $\mathrm{H} \alpha$ velocities (measured with the mirror method) folded with the best-fit $2.5 \mathrm{~d}$ ephemeris of V782 Cas. For $R V s$, filled points correspond to TIGRE data and the open circles to CARMENES data - note their good agreement.
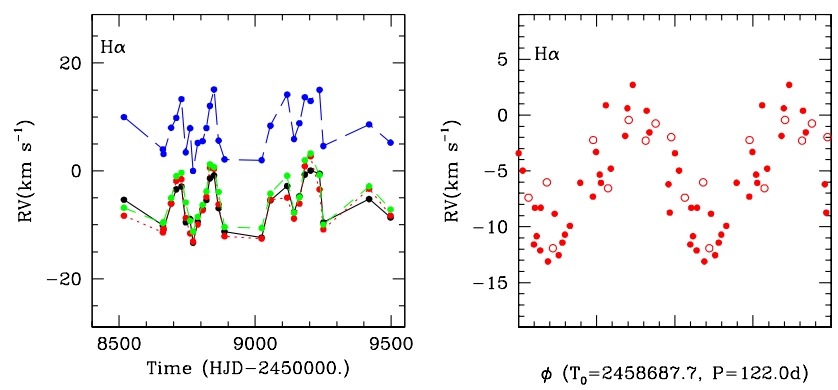

Figure 6. Left: Same as left and middle panels of Fig. 5 but for the $\mathrm{H} \alpha$ velocities. Right: $\mathrm{H} \alpha$ velocities (measured with the mirror method) folded with the best-fit $122 \mathrm{~d}$ ephemeris. Filled points correspond to TIGRE data and the open circles to CARMENES data.

Table 2. SB1 orbital solution of the short-period binary in V782 Cas as derived from the average $R V s$ of the He I lines obtained via spectral disentangling.

\begin{tabular}{cc}
\hline Parameter & Value \\
\hline $\mathrm{P}_{\text {orb }}($ days $)$ & 2.5132 \\
$e$ & 0 \\
$v_{0}\left(\mathrm{~km} \mathrm{~s}^{-1}\right)$ & $-15.5 \pm 1.2$ \\
$K\left(\mathrm{~km} \mathrm{~s}^{-1}\right)$ & $68.2 \pm 1.9$ \\
$a \sin i\left(\mathrm{R}_{\odot}\right)$ & $3.38 \pm 0.10$ \\
$T_{0}(\mathrm{HJD}-2450000)$ & $8516.648 \pm 0.009$ \\
$f(m)\left(\mathrm{M}_{\odot}\right)$ & $0.082 \pm 0.007$ \\
\hline
\end{tabular}

the method fails to distinguish the faint signature of the components of the short-period binary from the intrinsically variable contribution of the Be star. This is especially true for the regions around the Balmer lines. Significantly better results are obtained in the vicinity of the above-mentioned He I lines, where we managed to reconstruct bits of the spectrum of the primary of the short-period binary. In the spectral disentangling procedure, the $R V s$ of the $\mathrm{He}_{\mathrm{I}}$ lines inferred from the correlation method were used as input and updated $R V s$ were

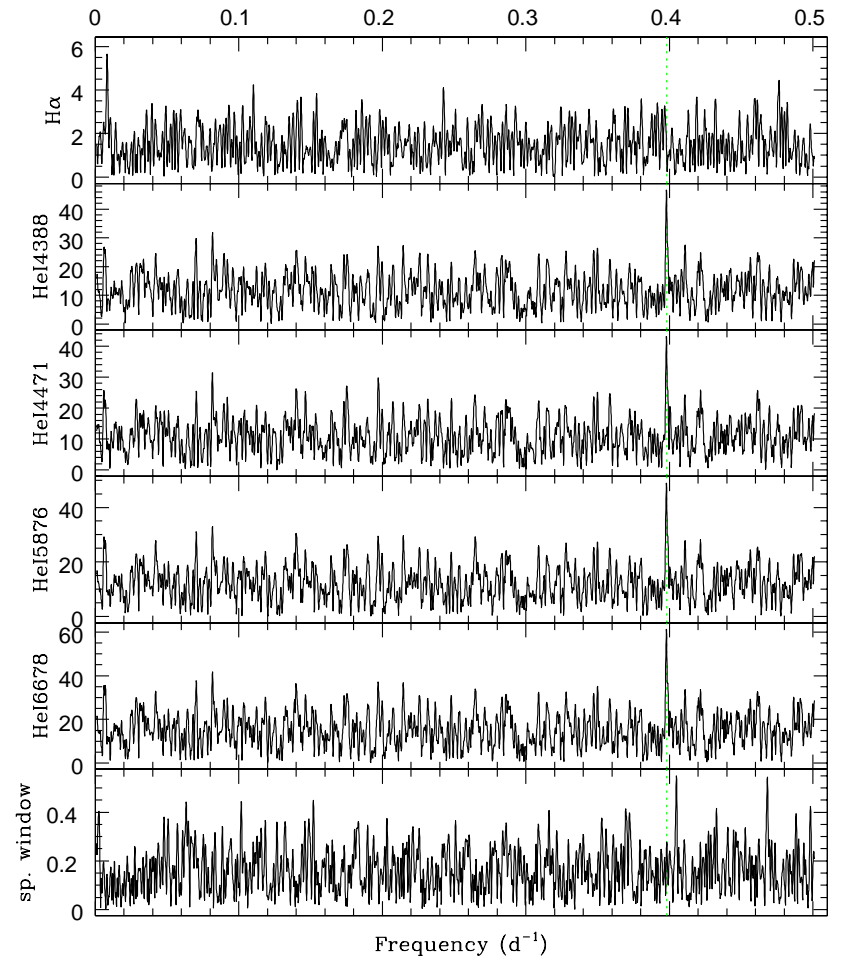

Figure 7. Periodogram derived for He I velocities (obtained by the correlation method on TIGRE data) and for $\mathrm{H} \alpha$ velocities (measured with the mirror method on TIGRE data) of V782 Cas by the modified Fourier algorithm, along with its spectral window. The dotted green line indicates the photometric frequency (Nazé et al. 2020c).

established by cross-correlation with a synthetic TLUSTY spectrum for a B2 V star (Lanz \& Hubeny 2003). Using these updated $R V s$, we derived the SB1 orbital solution shown on top of Fig. 8 and in Table 2.

Comparing the reconstructed $\mathrm{He}_{\mathrm{I}}$ lines with TLUSTY spectra for 

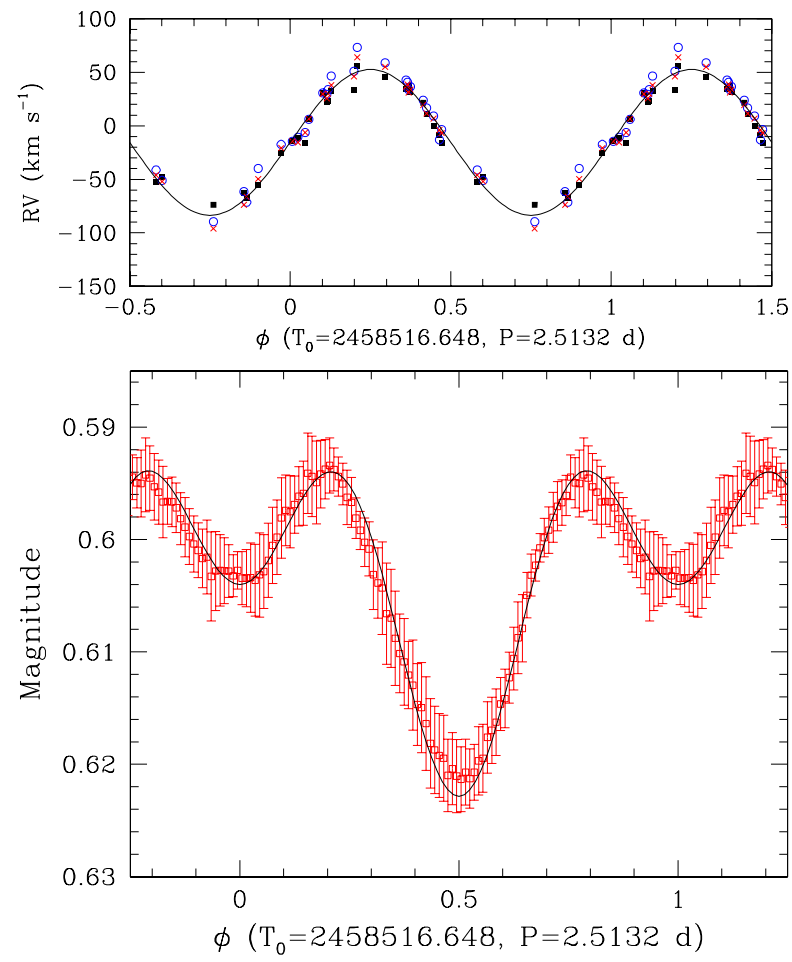

Figure 8. Top: SB1 orbital solution obtained from the $R V s$ of the $\mathrm{He}$ I absorption lines of V782 Cas inferred via the spectral disentangling method. Filled squares, open circles and red crosses stand for the $R V s$ of the He I 4713, 5876, and 6678 $\AA$ lines, respectively. Bottom: Best-fit Nightfall binary model fit of the normal lightcurve of V782 Cas built from the TESS data.

different projected rotational velocities, we found the best agreement for $v_{p} \sin i=75 \pm 10 \mathrm{~km} \mathrm{~s}^{-1}$. Beside the three He $\mathrm{I}$ lines used for the RV determination, the spectral disentangling unveiled absorption lines due to $\mathrm{He}_{\mathrm{I}} \lambda \lambda 4009,4026,4121,4144,4169,4388,4471 \AA$, C II $\lambda 4267 \AA$, Si III $\lambda \lambda 4552,4568 \AA$, and O II $\lambda 4649 \AA$. All these features were recovered with a strength consistent with a B2 spectral type classification diluted by a factor 0.28 , i.e. the B2 component contributes $28 \%$ of the total light of V782 Cas. The CARMENES spectra are of higher spectral resolution, but are less numerous, rendering any attempt to apply the disentangling method to the $\mathrm{He}_{\mathrm{I}}$ lines hopeless. Nevertheless, these data unveil a weak He i $\lambda 5876$ signature of the secondary of the short-period binary on three occasions. We deblended the primary and secondary contributions via a simultaneous fit of two Gaussians. Though the results of this fit must be considered with caution, the $R V s$ found for the primary nicely confirm the SB1 orbital solution inferred from the TIGRE data while the $R V s$ of the secondary suggest a value of $q=\frac{m_{p}}{m_{s}}$ between 1.3 and 1.7. Finally, the equivalent width of the secondary line appears to be about $1 / 4$ of that of the primary. This suggests that the secondary $\mathrm{He}_{\mathrm{I}}$ lines are diluted by about a factor $0.10-0.15$, i.e. the component contributes $10-15 \%$ of the total light of V782 Cas. This rules out the possibility considered by Nazé et al. (2020c) that the short-period binary might correspond to one of the neighbouring sources resolved by GAIA, as these objects would contribute much less than $\sim 40 \%$ to the combined light.

Since the TESS lightcurve of V782 Cas indicates an eclipsing binary in an overcontact configuration (see Nazé et al. 2020c, and below), the stars in the short-period binary must be in co-rotation. We can then use the formula of Eggleton (1983) to express the radius of

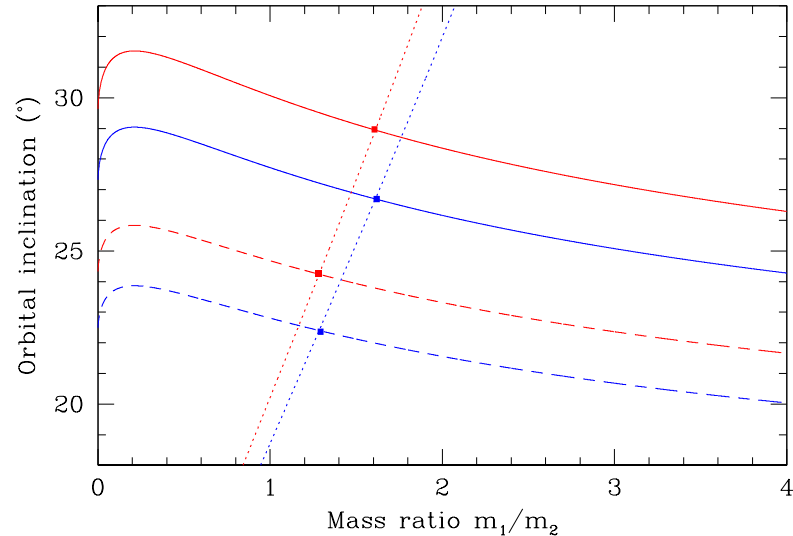

Figure 9. Constraints on $i$ and $q$ from the projected rotational velocity (Eq. 3) and the mass function (Eq. 5) of the primary star of the short-period binary in V782 Cas. The red and blue curves correspond to the results obtained for a primary mass of 8 or $10 \mathrm{M}_{\odot}$. The continuous and dashed lines show the results for Eq. 3 for $f i l l_{p}=1.0$ and $f i l l_{p}=1.2$, respectively. The dotted lines stand for the relation 5 obtained from the mass function.

a star (over)filling its Roche lobe as a function of the mass-ratio $q$, and from there its projected rotational velocity:

$v_{p} \sin i=\left(G m_{p}\right)^{1 / 3}\left(\frac{2 \pi}{P_{\text {orb }}}\right)^{1 / 3} \frac{0.49 q^{2 / 3}\left(1+\frac{1}{q}\right)^{1 / 3} f_{i l l}}{0.6 q^{2 / 3}+\ln \left(1+q^{1 / 3}\right)} \sin i$

where fill $_{p}$ is the Roche lobe filling factor of the primary. With $P_{\text {orb }}=2.5132$ days, this leads to

$v_{p} \sin i=76.6$ fill $_{p} \frac{q^{2 / 3}\left(1+\frac{1}{q}\right)^{1 / 3}}{0.6 q^{2 / 3}+\ln \left(1+q^{1 / 3}\right)}\left(\frac{m_{p}}{\mathrm{M}_{\odot}}\right)^{1 / 3} \sin i$

where the numerical factor is expressed in $\mathrm{km} \mathrm{s}^{-1}$.

Comparing this expression with the observed $v_{p} \sin i$ then allows us to express a relation between orbital inclination and mass-ratio:

$\sin i=0.978 \frac{0.6 q^{2 / 3}+\ln \left(1+q^{1 / 3}\right)}{f i l l_{p} q^{2 / 3}\left(1+\frac{1}{q}\right)^{1 / 3}}\left(\frac{m_{p}}{\mathrm{M}_{\odot}}\right)^{-1 / 3}$

This relation is displayed in Fig. 9 for two different assumptions on $m_{p}\left(8\right.$ and $\left.10 \mathrm{M}_{\odot}\right)$ and two different values of fill $_{p}(1.0$ and 1.20).

The mass function of the SB1 solution (Table 2) can be written:

$\frac{m_{s}^{3} \sin ^{3} i}{\left(m_{p}+m_{s}\right)^{2}}=\frac{P_{\text {orb }} K_{p}^{3}}{2 \pi G}\left(1-e^{2}\right)^{3 / 2}$

which, given that $e=0.0$, leads to

$\sin i=6.3810^{-3} K_{p}\left(\frac{\mathrm{M}_{\odot}}{m_{p}} q(1+q)^{2}\right)^{1 / 3}$

where $K_{p}$ is expressed in $\mathrm{km} \mathrm{s}^{-1}$. This relation is also displayed in Fig. 9, again for $m_{p}$ of 8 or $10 \mathrm{M}_{\odot}$. The intersection between the curves corresponding to relations 3 and 5 indicate that the mass ratio of the short-period binary is likely close to 1.5 , and that the orbital inclination is expected to be in the range between $22^{\circ}$ and $29^{\circ}$. Therefore, the secondary component of the short-period binary likely has a mass of $6_{-1.2}^{+1.5} \mathrm{M}_{\odot}$, which would correspond to an B5 star with an uncertainty of one or two spectral substypes. 


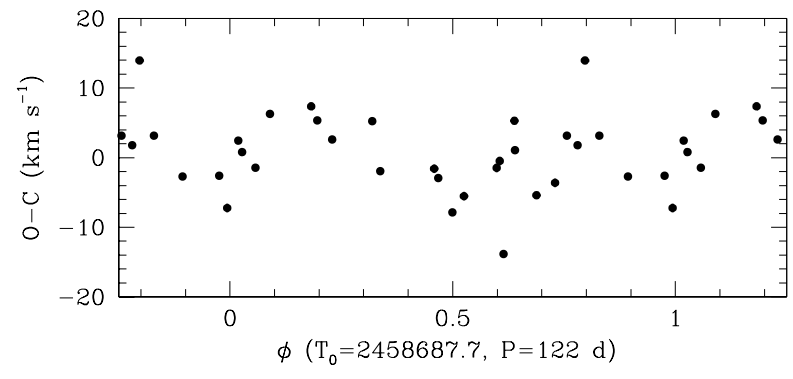

Figure 10. O-C residuals of the SB1 solution of the primary component of the short-period binary in V782 Cas folded with the ephemerides of the Be star.

We also revisited the analysis of the photometric lightcurve presented by Nazé et al. (2020c), taking advantage of the better knowledge of the short-period binary. Adopting the improved ephemerides of the short-period binary (Table 2$)^{4}$, we built an updated lightcurve consisting of 100 normal points computed as the mean magnitude per 0.01 phase bin. The dispersion of the data points within a phase bin is adopted as our estimate of the uncertainty. The Nightfall binary star code (Wichmann 2011) is then used to fit the lightcurve. The model accounts for the presence of third light, in this case coming from the Be star, and for reflection effects between the stars. We fixed the mass ratio to 1.5 and the primary star effective temperature to $20900 \mathrm{~K}$. We explored a grid of values of the Roche-lobe filling factors between 1.00 and 1.20, and third light contributions ranging from $57 \%$ to $62 \%$. There is a huge degeneracy between different models in the parameter space, but overall the lightcurve analysis confirms the orbital inclination to be in the $21^{\circ}-30^{\circ}$ range. The formally best solution for $q=1.5$ (i.e. masses of 9 and $6 M_{\odot}$ ) is found for fill $_{p}=$ fill $_{s}=1.175$, polar radii of 8.7 and $7.5 \mathrm{R}_{\odot}$, a third light contribution of $57 \%$ (corresponding to V782 Cas A), an orbital inclination of $21.5^{\circ}$ and a secondary mean temperature (accounting for the heating due to reflection effects) of $11710 \mathrm{~K}$. In this solution, the primary star contributes $35 \%$ of the total light of V782 Cas, whereas the secondary provides $8 \%$ of the light. Those values agree well with those found from spectroscopy (see above). The lightcurve and its best-fit model are shown at the bottom of Fig. 8. Because of the low inclination, the recorded variations are not true eclipses. Rather, the photometric changes are due to ellipsoidal variations. The unequal depth of the photometric minima is due to the difference in surface brightness distribution of the two stars, which is notably affected by reflection effects. Whilst the overall quality of the fit is rather good, some of the remaining deviations are likely due to the intrinsic variability of the Be star (Nazé et al. 2020c). The light ratio of about 4 between the two stars in V782 Cas B agree well with their respective spectral types, but we note that the temperature of the secondary object is a little bit low for the spectral type of B5 mentioned above (Drilling \& Landolt 2000). However, because of the on-going interaction (both stars overfill their Roche lobes), the stars may present non-standard parameters.

\footnotetext{
${ }^{4}$ Note that an incorrect $T_{0}$ was mentioned in Nazé et al. (2020c): the actual value to get Fig. A1 in that paper (with $\phi=0$ for the main eclipse) was 2458789.349 . However, the new data now provide better ephemeris so that the old values can now be dismissed.
}

\subsubsection{V782 Cas A, the long-period binary}

Turning to the long-period binary, we fitted the $R V s$ of the $\mathrm{H} \alpha$ line by a sinusoid, resulting in an amplitude of $5.2 \pm 0.9 \mathrm{~km} \mathrm{~s}^{-1}$ with $T_{0}=2458687.7 \pm 2.6$ (conjunction with the Be star in front). The velocity dispersion then decreases from $4.4 \mathrm{~km} \mathrm{~s}^{-1}$ to $2.6 \mathrm{~km} \mathrm{~s}^{-1}$ (see right panel of Fig. 6 - note the very good agreement between CARMENES and TIGRE data). The associated mass function is here $0.0018 \pm 0.0009 M_{\odot}$. Considering the Be star (spectral type B2.5III) to have a mass of $9 M_{\odot}$ and the system's inclination to be $60-90^{\circ}$, the companion should then have a mass of $0.55-0.65 \pm 0.10 M_{\odot}$. The period is similar to that found for the $\phi$ Per system (Mourard et al. 2015 ) but the amplitude is half in V782 Cas. Hence the companion is less heavy, relative to the primary star and for a similar inclination. Note that V782 Cas has not been studied by Wang et al. (2018).

To check whether the short-period binary and the Be star are gravitationally bound with an orbital period of $122 \mathrm{~d}$, we folded the $\mathrm{O}-\mathrm{C}$ residuals of the above SB1 solution of the short-period binary with the ephemerides of the Be star. The result is illustrated in Fig. 10. Whilst we observe $\mathrm{O}-\mathrm{C}$ values that range between -14 and $+14 \mathrm{~km} \mathrm{~s}^{-1}$, there is no clear trend that would indicate a reflex motion with respect to the Be star. This suggests that the period of the Be star is not an orbital period around the short-period binary, but rather around another, unseen companion.

\subsection{HD 45995}

HD 45995 was noted to have a companion with common proper motion, lying at $16.1^{\prime \prime}$ from the star, in Abt \& Cardona (1984). Its presence is confirmed in Gaia-DR3 although nothing is mentioned about it in Mason et al. (1999); Roberts et al. (2007); Mason et al. (2009); Maíz Apellániz (2010); Horch et al. (2020). Large $\left(50 \mathrm{~km} \mathrm{~s}^{-1}\right)$ changes in radial velocity were reported by Harmanec (1987). He pointed to a period shorter than $10 \mathrm{~d}$, preferentially $5.29 \mathrm{~d}$. Similar changes were also detected by Gies \& Bolton (1986), but they explained them in terms of non-radial pulsations of period $1.23 \mathrm{~d}$. Our analysis of TESS photometry detected the presence of a frequency of $1.184 \mathrm{~d}^{-1}$ (Nazé et al. 2020c), of which the $1.23 \mathrm{~d}$ period is a daily alias.

In the TIGRE data, variations of the radial velocities seem to be present but no obvious timescale is readily seen by eye (Fig. 11). We nevertheless calculated the periodogram associated to the $\mathrm{H} \alpha$ velocities (derived with the mirror method). It reveals a peak for a period of $103.1 \pm 1.0 \mathrm{~d}$, with an amplitude triple of the mean level of the periodogram (Fig. 12). Folding the velocities with this period yields a very convincing result (Fig. 13). It must be noted that a clear sinusoidal modulation is observed for both $\mathrm{H} \alpha$ and $\mathrm{H} \beta$, but also for $\mathrm{He}_{\mathrm{I}}$ lines although their RVs are noisier. A $\chi^{2}$ fit to $\mathrm{H} \alpha$ velocities yields a best-fit sinusoid with parameters: $T_{0}=2458575.5 \pm 0.8$ (conjunction with Be primary in front) and $K=6.7 \pm 0.4 \mathrm{~km} \mathrm{~s}^{-1}$. The $\mathrm{H} \alpha$ velocity dispersion passes from an initial $5 \mathrm{~km} \mathrm{~s}^{-1}$ to only $1.5 \mathrm{~km} \mathrm{~s}^{-1}$ after that best-fit sinusoid is taken out. The best-fit parameters yield a mass function of $0.0032 \pm 0.0006 M_{\odot}$. Considering the Be star (spectral type B2V) to have a mass of $10 M_{\odot}$ (Vieira et al. 2017) and the system's inclination to be $46.8^{\circ}$, i.e. that of the disk (Frémat et al. $2005)$, the companion should then have a mass of $1.0 \pm 0.1 M_{\odot}$.

\subsection{HD 44458}

This star is most probably associated with a 4 " neighbouring star (Abt \& Cardona 1984; Roberts et al. 2007). In the latter reference, the companion is said to have a spectral type B8-A0 V, with the Be 

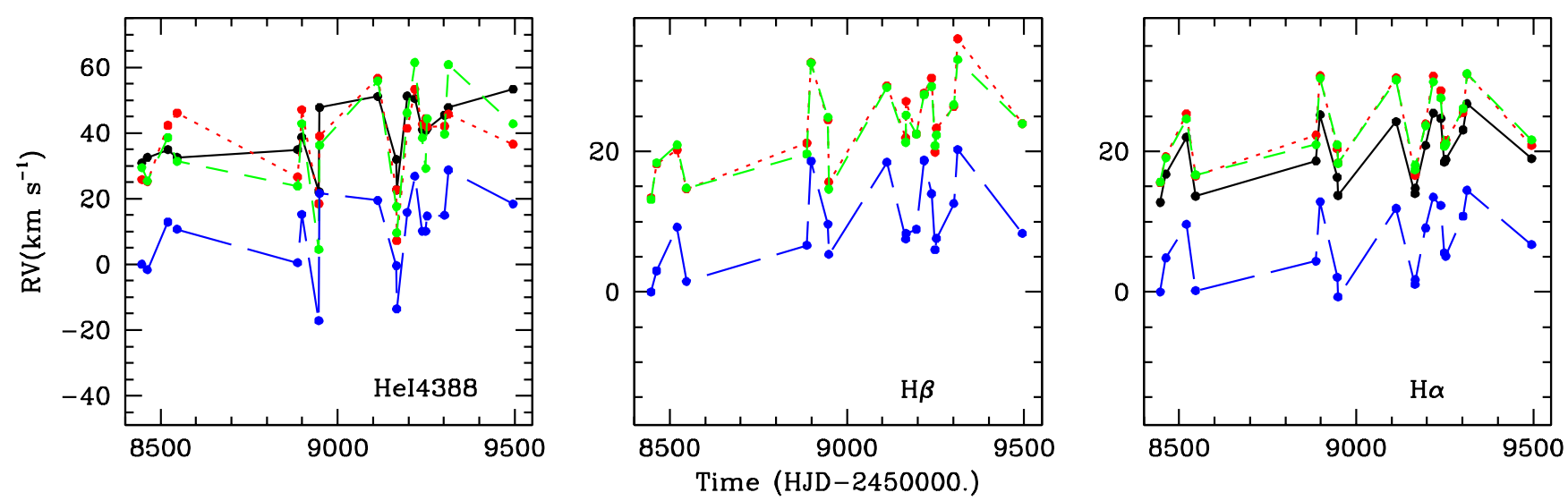

Figure 11. Radial velocities measured for HD 45995 on $\mathrm{He}$ I $4388 \AA$ (left), $\mathrm{H} \beta$ (middle) and $\mathrm{H} \alpha$ (right) lines using the moment method (black points and solid line), the mirror method (red points and dotted line), the double Gaussian method (green points and dashed line), and the correlation method (blue points and long dashed line). Note that the mix of absorption and emission in $\mathrm{H} \beta$ makes the first order moments unreliable hence the associated RVs are not shown.

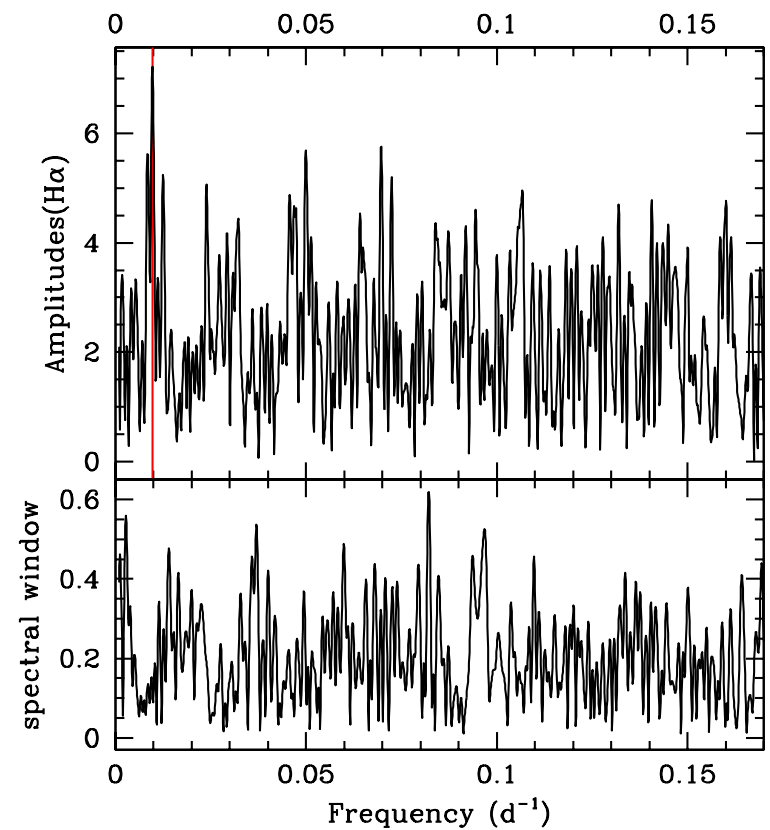

Figure 12. Periodogram derived with the modified Fourier algorithm for $\mathrm{H} \alpha$ velocities of HD 45995 (measured with the mirror method), along with its spectral window. A vertical line indicates the proposed orbital period.

star reported to be a Cepheid (!). No information about a closer companion is available, since no change in radial velocity was reported in literature. However, clear RV variations are seen in our data and they are relatively coherent from line to line (Fig. 14). Their amplitude is about $10 \mathrm{~km} \mathrm{~s}^{-1}$ in $\mathrm{H} \alpha$. While the velocities were analyzed with the different period search algorithms, no coherent picture emerges. Furthermore, the folding with the best-fit "period" found with the Fourier method (332 $\pm 10 \mathrm{~d}$ - suspiciously close to a year) is not totally convincing: the RVs are found to cover only two-third of the alleged orbit and to increase monotoneously in that interval. Therefore, while the presence of velocity variations seems ascertained and the star can be considered as a binary candidate, more observations

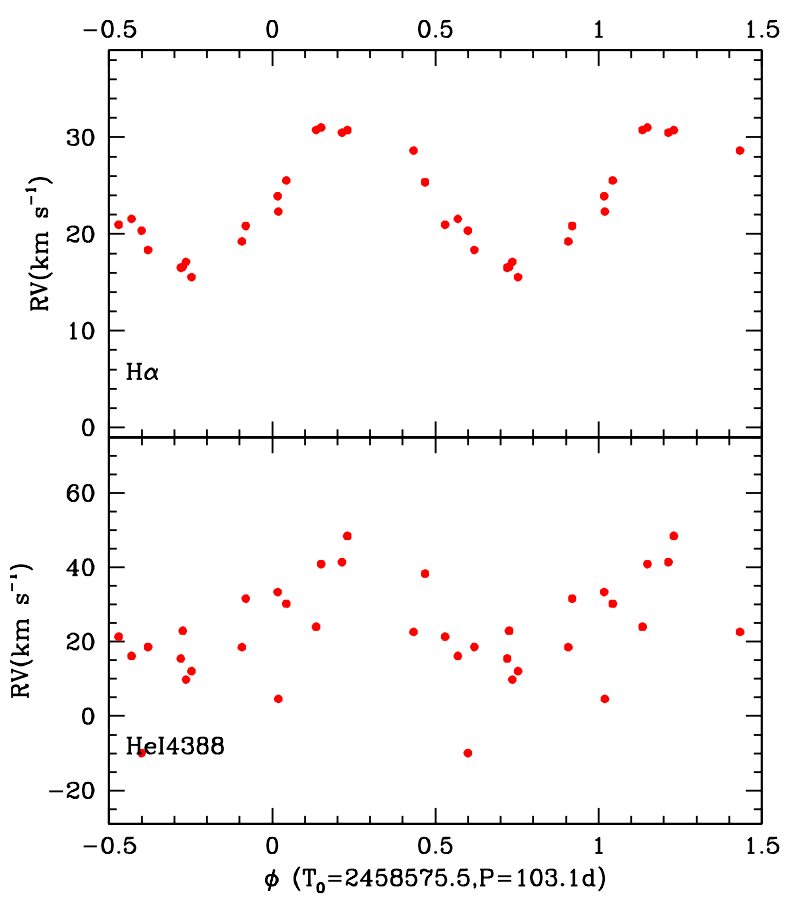

Figure 13. Velocities of HD 45995 measured with the mirror method for He I $4388 \AA$ (bottom) and $\mathrm{H} \alpha$ (top) folded with the best-fit ephemeris.

are needed before assessing their recurrence. We may however already conclude that any recurrence timescale must be larger than the yearly observing campaign durations (100-300 d).

\subsection{Other northern stars}

No optical companion was reported for SAO 49725, V2156 Cyg, and V810 Cas (Abt \& Cardona 1984; Mason et al. 1999; Roberts et al. 2007; Mason et al. 2009; Maíz Apellániz 2010; Horch et al. 2020). 

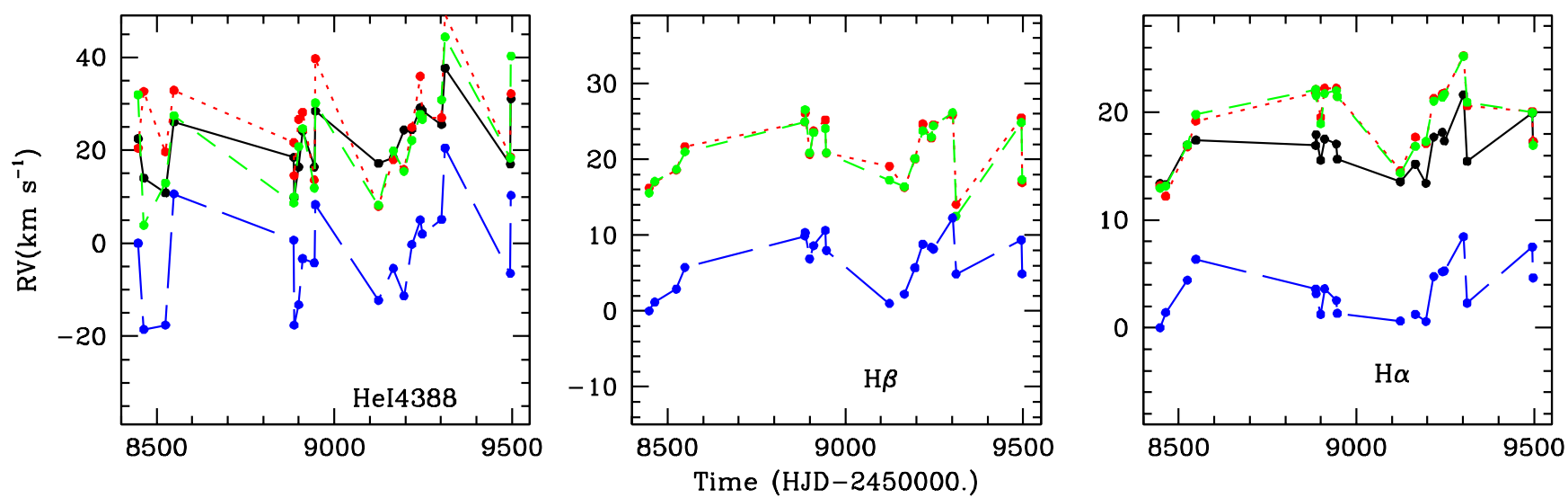

Figure 14. Radial velocities measured for $\mathrm{HD} 44458$ on $\mathrm{He}$ I $4388 \AA$ (left), $\mathrm{H} \beta$ (middle) and $\mathrm{H} \alpha$ (right) lines using the moment method (black points and solid line), the mirror method (red points and dotted line), the double Gaussian method (green points and dashed line), and the correlation method (blue points and long dashed line). Note that the mix of absorption and emission in $\mathrm{H} \beta$ makes the first order moments unreliable hence they are not shown.
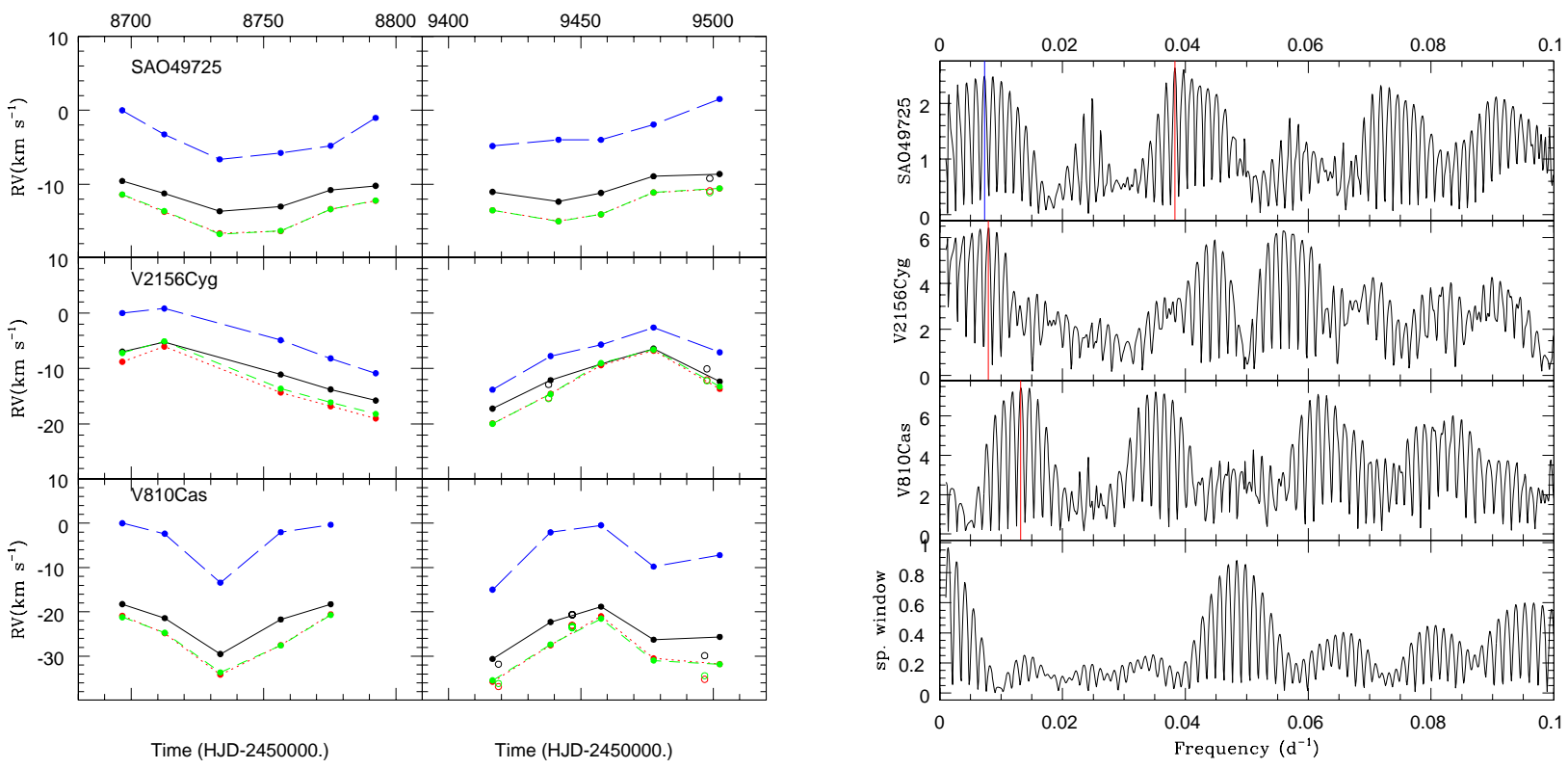

Figure 15. Left: Velocities measured in 2019 (left) and 2021 (right) for SAO 49725 (top), V2156 Cyg (middle), and V810 Cas (bottom). These velocities were derived from the $\mathrm{H} \alpha$ lines using the moment method (black points and solid line), the mirror method (red points and dotted line), the double Gaussian method (green points and dashed line), and the correlation method (blue points and long dashed line). Filled points correspond to velocities derived from CARMENES observations, open symbols from TIGRE observations. Right: Periodograms derived from the CARMENES velocities using the mirror method. Vertical red and blue lines indicate the highest peaks chosen as orbital periods.

The CARMENES data of these three stars display clear RV variations, and these velocity changes agree for all measurements methods and for all considered lines ( $\mathrm{H}, \mathrm{He}, \mathrm{Fe})$. This is clearly reminiscent of binary motion. The velocity changes observed in 2019 were confirmed in the 2021 campaign (left panels of Fig. 15). The number of velocity measurements $(\sim 10)$ is sufficient to attempt a period search (right panels of Fig. 15). While some peaks can be detected, with an amplitude of about 2.5 times the mean level of the periodogram, numerous aliases are also present. For SAO 49725, this even leads to two equally high peaks, corresponding to two plausible periods and even the inclusion of TIGRE data does not allow to distinguish them. Therefore the derived periods require confirmation. Nevertheless, as for the previous cases, we have derived the best-fit sinusoidal solutions using a $\chi^{2}$ fitting.

For SAO 49725, the two preferred periods are $26.11 \pm 0.08 \mathrm{~d}$ and $137.0 \pm 2.3 \mathrm{~d}$. The former value leads to an amplitude of $2.8 \pm 0.5 \mathrm{~km} \mathrm{~s}^{-1}$, which in turn yields a mass function of $(5.9 \pm 3.2) \times 10^{-5} \mathrm{M}_{\odot}$, and $T_{0}=2458712.1 \pm 0.3$. For the latter values, one rather finds $K=2.7 \pm 0.7 \mathrm{~km} \mathrm{~s}^{-1}, T_{0}=2458780.0 \pm 1.5$, and $f(m)=(2.8 \pm 2.2) \times 10^{-4} \mathrm{M}_{\odot}$. In both cases, the velocity disper- 
Table 3. Results from our velocity monitoring. For confirmed binaries, the period, amplitude, and zero-points of their $R V$ curves are provided; for binary candidates, approximate values for the temporal interval between the most distant exposures and for the maximum $R V$ shift are noted. The number of measurements $N$ is also listed, and the two possibilities for SAO 49725 are quoted. $\gamma$ values depend on the method used: listed values are those found for velocities derived by the mirror method.

\begin{tabular}{|c|c|c|c|c|c|}
\hline \multicolumn{6}{|c|}{ Confirmed binaries } \\
\hline Name & $N$ & $\begin{array}{l}P \\
\text { (d) }\end{array}$ & $\begin{array}{c}K \\
\left(\mathrm{~km} \mathrm{~s}^{-1}\right)\end{array}$ & $\begin{array}{c}\gamma \\
\left(\mathrm{km} \mathrm{s}^{-1}\right)\end{array}$ & $\begin{array}{c}T_{0} \\
\text { (HJD-2 450 000) }\end{array}$ \\
\hline V782 CasA & $27+9$ & $122.0 \pm 1.5$ & $5.2 \pm 0.9$ & $-5.6 \pm 0.2$ & $8687.7 \pm 2.6$ \\
\hline HD 45995 & 19 & $103.1 \pm 1.0$ & $6.7 \pm 0.4$ & $23.9 \pm 0.2$ & $8575.5 \pm 0.8$ \\
\hline V558 Lyr & 14 & $83.3 \pm 1.8$ & $8.2 \pm 1.1$ & $-46.9 \pm 0.3$ & $9056.2 \pm 1.4$ \\
\hline \multirow[t]{2}{*}{ SAO 49725} & $11+1$ & $26.11 \pm 0.08$ & $2.8 \pm 0.5$ & $-13.7 \pm 0.3$ & $8712.1 \pm 0.3$ \\
\hline & & $137.0 \pm 2.3$ & $2.7 \pm 0.7$ & $-12.1 \pm 0.3$ & $8780.0 \pm 1.5$ \\
\hline V2156 Cyg & $10+3$ & $126.6 \pm 2.0$ & $5.5 \pm 0.7$ & $-12.9 \pm 0.3$ & $8812.0 \pm 1.2$ \\
\hline V810 Cas & $10+3$ & $75.8 \pm 0.7$ & $6.4 \pm 0.7$ & $-27.9 \pm 0.3$ & $8753.0 \pm 2.0$ \\
\hline \multicolumn{6}{|c|}{ Binary candidates } \\
\hline Name & $N$ & $\begin{array}{l}\Delta(t) \\
\text { (d) }\end{array}$ & $\begin{array}{l}\Delta(R V) \\
\left(\mathrm{km} \mathrm{s}^{-1}\right)\end{array}$ & & \\
\hline HD 44458 & 20 & 140 & 10 & & \\
\hline HD 110432 & 6 & 70 & 10 & & \\
\hline HD 119682 & 6 & 90 & 20 & & \\
\hline HD 161103 & 5 & 160 & 10 & & \\
\hline V771 Sgr & 7 & 170 & 15 & & \\
\hline
\end{tabular}
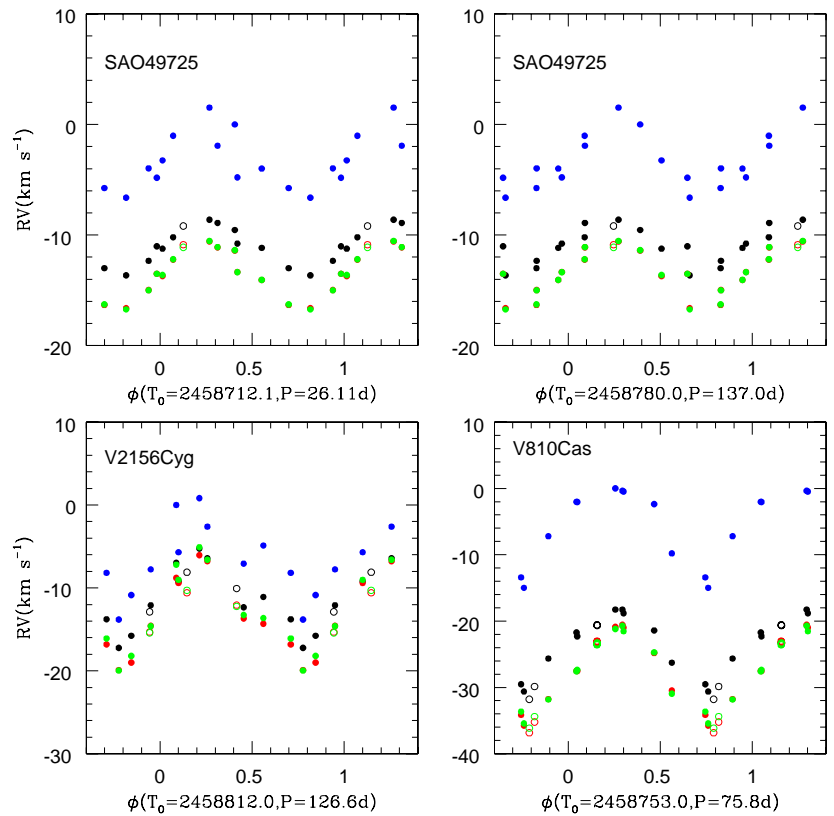

Figure 16. Same velocities as in left panels of Fig. 15, but phase-folded with the best-fit ephemeris.

sions changes from 2 to $1 \mathrm{~km} \mathrm{~s}^{-1}$ after the fitting. The star displays rather narrow lines, compared to other stars in this sample, suggesting that it is viewed under a lower inclination. Hence we enlarged the considered range of orbital inclinations to $30-90^{\circ}$, and then find secondary masses of $0.22-0.45 \pm 0.05 \mathrm{M}_{\odot}$ and $0.4-0.7 \pm 0.1 \mathrm{M}_{\odot}$, respectively, considering a mass for the Be star intermediate between those of $\gamma$ Cas and $\pi$ Aqr (see below).
For V2156 Cyg, Chojnowski et al. (2017) reported RV variations from -20 to $-5 \mathrm{~km} \mathrm{~s}^{-1}$ on the Br11 hydrogen line at $1.68 \mu \mathrm{m}$. While their dataset consisted of only three spectra, the presence of velocity variations is confirmed by our data. Our periodogram presents its strongest peak at $126.6 \pm 2.0 \mathrm{~d}$. The $\chi^{2}$ fit to $\mathrm{H} \alpha$ velocities yields a best-fit sinusoid with parameters $T_{0}=2458812.0 \pm 1.2$ (conjunction with Be primary in front) and $K=5.5 \pm 0.7 \mathrm{~km} \mathrm{~s}^{-1}$. The velocity dispersion passes from an initial $5 \mathrm{~km} \mathrm{~s}^{-1}$ to only $2 \mathrm{~km} \mathrm{~s}^{-1}$ after that best-fit sinusoid is taken out. These best-fit parameters yield a mass function of $0.0022 \pm 0.0008 \mathrm{M}_{\odot}$. As the Be star has a spectral type of B1.52V, we may assume its mass to be about $11 \mathrm{M}_{\odot}$ (Vieira et al. 2017). With a system's inclination in the range 60 to $90^{\circ}$, this leads to a companion mass of $0.7-0.8 \pm 0.1 M_{\odot}$.

For V810 Cas, the strongest peak is at $75.8 \pm 0.7 \mathrm{~d}$ and the sinusoid fitting to the $\mathrm{H} \alpha$ velocities yields $K=6.4 \pm 0.7 \mathrm{~km} \mathrm{~s}^{-1}$ and $T_{0}=$ $2458753.0 \pm 2.0$, with a dispersion decreased from 5 to $1 \mathrm{~km} \mathrm{~s}^{-1}$. With a spectral type B1 (hence a mass of about $12.5 \mathrm{M}_{\odot}$, Vieira et al. 2017), we find $f(m)=0.0021 \pm 0.0007 \mathrm{M}_{\odot}$ and a secondary mass of $0.7-0.8 \pm 0.1 M_{\odot}$ for an inclination range of $60-90^{\circ}$.

These solutions provide convincing phase plots (right panels of Fig. 16). However, as mentioned above, all these orbital solutions should be considered as preliminary because of the aliases in the periodogram. More data should be collected to confirm them in the near future. It is however important to note that the few existing TIGRE measurements are fully consistent with the CARMENES data (Fig. 16).

\subsection{Southern stars}

The southern sample suffered from a lack of multiplicity investigation. Only V767 Cen was mentioned to have a distant optical companion (a B8V star at 15", Lindroos 1985), while high-resolution interferometric data could not detect any companion to HD 110432 (Stee et al. 2013; Sana et al. 2014). In our UVES data, all stars display velocity changes (Fig. 17). However, the line profiles also appear much more variable than for TIGRE and CARMENES targets, which could feign velocity changes unrelated to binarity. Besides, the detected trends are not always consistent amongst different lines, pointing to another probable cause than binarity. Therefore, we split the results in different categories.

Both HD 157832 and HD 316568 display prominent line profile variations, both in He I absorptions and in the core of Balmer emissions. For example, one of the He I profiles of HD 157832 appears perfectly triangular while it is rounded at other dates, whereas the $\mathrm{He}$ I profiles of HD 316568 appear more or less skewed or with a flat bottom. This points towards pulsational activity, which is known to exist in HD 157832 from photometric data (Nazé et al. 2020c). Therefore, we cannot attribute the observed velocity changes to binarity with any certainty.

Some stars rather display significant changes in overall disk activity. The first observation of V767 Cen, taken three months before the four other ones, revealed a much reduced strength of the disk emissions. Discarding this outlier and focusing only on the last data, the velocities vary in a negligible way, showing no obvious binary signature. There are also clear changes in the disk emissions of HD 119682, with an increased activity in the last observation. However, the line profiles of $\mathrm{He}_{\text {I }} \lambda 4026,4143,4388 \AA$ appear quite stable and their velocities display coherent trends. We therefore consider these observations as tentative evidence for binary motion.

For HD 90563, the measured velocities do not agree well between methods and/or between lines. For CQ Cir, the mesurement methods agree for a given line but vary from line to line. This renders those 
Hel4143
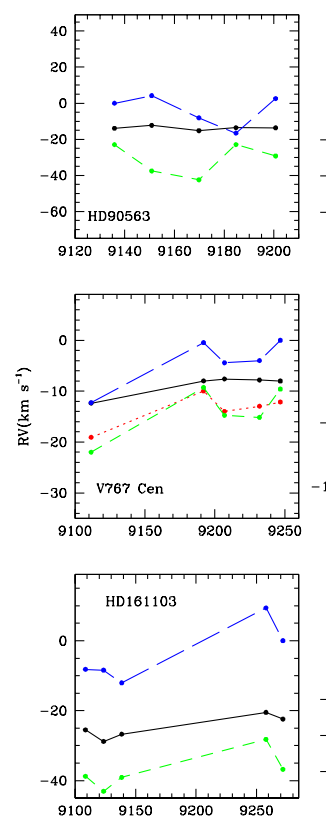
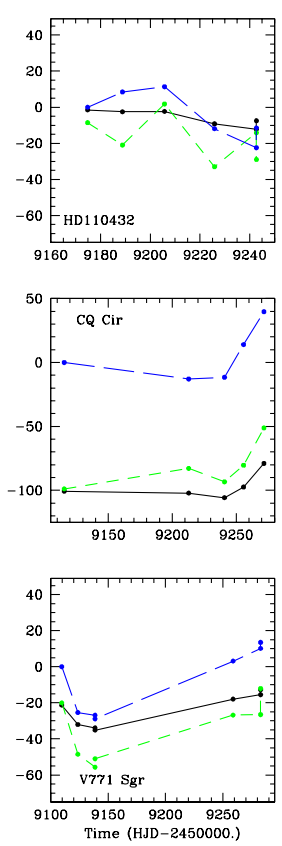
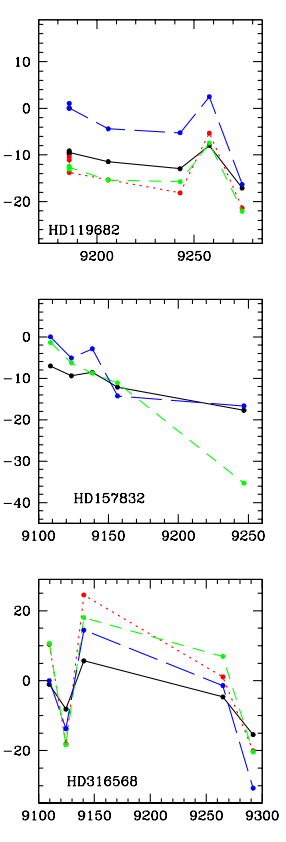

$\mathrm{H} \beta$
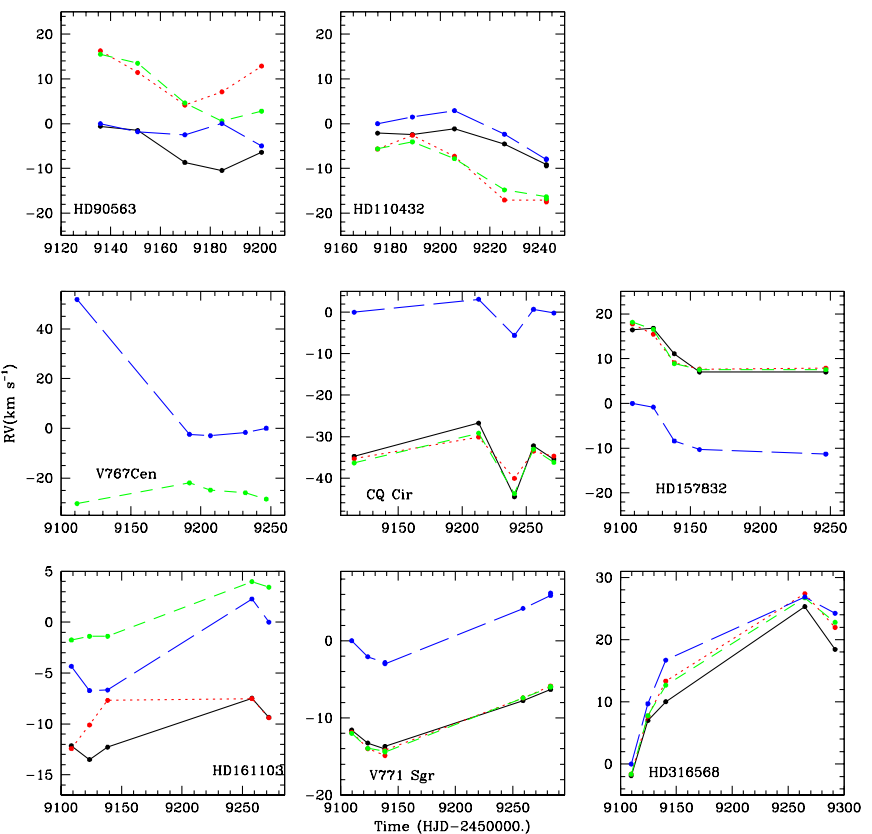

Figure 17. Velocities of the He $\mathrm{r} \lambda 4143 \AA$ line (left panels) and $\mathrm{H} \beta$ line (right panels) recorded in the southern targets observed by UVES. Symbols are as in Fig. 15. Four cases (HD 110432, HD 119682, HD 161103, and V771 Sgr) are kept as binary candidates.

stars unsuitable for entering a list of binary candidates, although more observations would be welcome to shed some light on the behaviour of these targets.

Coherent velocity changes between lines and between measurement methods are found for HD 110432, HD 161103, and V771 Sgr, however. The relatively long variation timescales and the shallow amplitudes are reminiscent of what is seen in the known binaries ( $\gamma$ Cas, $\pi$ Aqr, and the three new systems found in previous sections).

While evidence for binarity in the southern sample observed by UVES remains sparse so far, we find four cases (HD 110432, HD 119682, HD 161103, and V771 Sgr) showing promising hints. Therefore we grant those stars the status of binary candidates. As for CARMENES targets, further observations are required to confirm their multiplicity status and establish orbital solutions.

\section{DISCUSSION}

Our monitoring campaign of $\gamma$ Cas stars has revealed velocity changes in all stars, but not all of them could be securely attributed to binary motions. Amongst the 16 studied stars, a binary orbit could be well established for the Be star in three cases, a further three cases provided preliminary orbits, and five other stars can be considered as binary candidates (Table 3 ). Adding the two known binaries ( $\gamma$ Cas, $\pi$ Aqr), this means that the velocity of $72 \%$ of known $\gamma$ Cas stars has been monitored. Eight out of 18 (half of them) display definitive signatures of binary motion, with periods and amplitudes of orbital solutions ascertained. When the binary candidates are added, nearly three quarters of the studied stars are or could be binaries. The binary incidence amongst $\gamma$ Cas stars thus appears far from negligible. However, this fraction might only reflect the incidence of binaries amongst classical Be stars (see Section 1.1), rather than being a specific feature of $\gamma$ Cas analogs.

In this context, it is important to examine the properties of orbits and companions of Be stars. To this aim, Table 4 summarizes the properties of known Be binary systems. The top of the table provides the information available for the $\gamma$ Cas stars while the bottom part lists cases with early-type (B0-B3) classical Be stars not known to belong to the $\gamma$ Cas category. Because they represent a different evolutionary stage, we have excluded here the currently interacting cases in which the Be star actively accretes material from a red giant companion (e.g. AX Mon, V644 Mon, CX Dra, V360 Lac - Elias et al. 1997; Aufdenberg 1994; Harmanec et al. 2015). Furthermore, the table only presents systems with well-defined orbital solutions. This differs from the choices of Bodensteiner et al. (2020) who examined Be stars with spectral type B1.5e or earlier and split the "systems" depending on the companion's nature (post-MS/unknown). These authors considered some ambiguous indirect information as secure binarity evidence ${ }^{5}$ while missing some secure binary cases such as the well-known $\phi$ Per system. Focusing on systems with existing orbital solutions, as done here, is certainly more restrictive but, in our opinion, it helps avoiding any ambiguity.

Table 4 clearly reveals some common features amongst Be binaries: periods are generally long (a few months ${ }^{6}$ ) and the eccentricities small. This is valid for the $\gamma$ Cas stars as for the other Be stars. Regarding the secondary mass, seven early Be have companion with

\footnotetext{
5 In Bodensteiner et al. (2020), HD 161103 (=V3892 Sgr) is categorized as having a "known post-MS companion" (their class i) while the provided references only offer a general discussion on the origin of the $\gamma$ Cas phenomenon, with the magnetic and WD hypotheses highlighted: none of the provided references provide a direct, secure detection of a companion. Our velocity monitoring can only attribute it a "binary candidate" status.

${ }^{6}$ Note that the period of $\gamma$ Cas is at the long period extreme of the period distribution in this Table, but this system went through intensive radial velocity searches by many authors. It thus may be expected that more extensive searches in other stars not yet known to be in binaries could ultimately lead to other long period cases.
} 
Table 4. List of binaries with an early (B0-3) classical Be primary and an existing orbital solution, ordered by right ascension (R.A.).

\begin{tabular}{lccccccc}
\hline \hline Name & $P$ & $e$ & Be Sp.type & $\begin{array}{c}M(\mathrm{Be}) \\
\left(\mathrm{M}_{\odot}\right)\end{array}$ & $\begin{array}{c}M_{\text {comp }} \\
\left(\mathrm{M}_{\odot}\right)\end{array}$ & $\begin{array}{c}i \\
\left({ }^{\circ}\right)\end{array}$ & Reference \\
\hline$\gamma$ Cas stars & & & & & & & \\
$\gamma$ Cas & 203.6 & 0 & B0IV & 13 & $0.98^{*}$ & 45 & Nemravová et al. (2012) \\
V782 Cas & 122.0 & 0 & B2.5III & 9 & $0.6-0.7^{*}$ & $60-90$ & this work \\
HD 45995 & 103.1 & 0 & B2V & 10 & $1.0 \pm 0.1^{*}$ & 46.8 & this work \\
V558 Lyr & 83.3 & 0 & B3V & 8 & $0.7-0.8^{*}$ & $60-90$ & this work \\
SAO 49725 & 26.11 & 0 & B0.5III & 13 & $0.2-0.5^{*}$ & $30-90$ & this work \\
& 137.0 & 0 & & & $0.4-0.7^{*}$ & $30-90$ & this work \\
V2156 Cyg & 126.6 & 0 & B1.5V & 11 & $0.7-0.8^{*}$ & $60-90$ & this work \\
$\pi$ Aqr & 84.1 & 0 & B1V & 15 & $2.4 \pm 0.5$ & 70 & Bjorkman et al. (2002) \\
V810 Cas & 75.8 & 0 & B1 & 12.5 & $0.7-0.8^{*}$ & $60-90$ & this work \\
\hline Other Be stars & & & & & & & \\
$\phi$ Per & 126.7 & 0 & B1.5V & 9.6 & $1.2 \pm 0.2$ & 77.6 & Mourard et al. (2015) \\
Y Tau & 133.0 & 0 & B1IV & 11 & $0.9-1.0^{*}$ & $60-90$ & Ruždjak et al. (2009) \\
HR 2142 & 80.9 & 0 & B1.5IV-V & 10.5 & $0.7^{*}$ & 85 & Peters et al. (2016) \\
LB-1 & 78.8 & 0 & B3V & $7 \pm 2$ & $1.5 \pm 0.4^{*}$ & 39 & Shenar et al. (2020) \\
HD 55606 & 93.8 & 0 & B2.5-3V & $6.0-6.6$ & $0.83-0.9$ & $75-85$ & Chojnowski et al. (2018) \\
FY CMa & 37.3 & 0 & B0.5IV & $10-13$ & $1.1-1.5$ & $>66$ & Peters et al. (2008) \\
o Pup & 28.9 & 0 & B1IV & $11-15$ & $0.7-1.0^{+}$ & & Koubský et al. (2012) \\
MX Pup & 5.15 & 0.46 & B1.5III & 15 & $0.6-6.6$ & $5-50$ & Carrier et al. (2002) \\
$\chi$ Oph & 138.8 & 0.44 & B2V & 10 & $1.7-2^{*}$ & $60-90$ & Abt \& Levy (1978) \\
HD 161306 & 99.9 & 0 & B0 & 15 & $0.9^{+}$ & & Koubský et al. (2014) \\
HR 6819 & 40.3 & 0.04 & B2.5V & 6 & $0.4-0.8^{*}$ & 35 & Gies \& Wang (2020) \\
59 Cyg & 28.2 & 0.14 & B1.5V & $6.3-9.4$ & $0.6-0.9$ & $60-80$ & Peters et al. (2013) \\
60 Cyg & 146.6 & 0 & B1V & 11.8 & $1.5-3.4$ & $>29$ & Koubský et al. (2000) \\
\hline
\end{tabular}

${ }^{*}$ indicates SB1 systems for which the secondary mass was estimated from the probable values of primary mass, mass function, and inclination. ${ }^{+}$indicates SB2 systems for which the secondary mass was estimated from the mass ratio (derived from velocity amplitudes) and a probable value of the primary mass. When unconstrained, inclination is taken to be $60-90^{\circ}$. Note that, for $\gamma$ Cas, the results of Smith et al. (2012) and Nemravová et al. (2012) are similar - only the latter is quoted here. Velocity shifts have also been recorded for the secondary in HD 194335, although a full orbital coverage has not yet been acquired (Wang et al. 2021) hence we do not yet add it to this Table.

masses below one solar mass, two with masses of $1-1.5 M_{\odot}$, and two with masses larger than $1.5 M_{\odot}$. For $\gamma$ Cas stars, the respective numbers in these categories are 5, 2, and one. There is thus a prevalence of low-mass $\left(<1.5 M_{\odot}\right)$ companions in the known systems.

What does it imply on the nature of the companions? Considering first non-degenerate companions, such masses are compatible with late-type main-sequence stars (F or later) as well as with stripped Helium stars. However, it would be difficult to explain a strict preference towards late-type main-sequence stars when $\mathrm{O}$ and B-type systems rather display a preference towards early-type companions, i.e. $q$ ratios tend to be just less than 1 (e.g. Sana et al. 2012). Also, such low-mass stars should not display the UV signatures detected for some of the systems listed at the bottom of Table 4; rather, this type of signature is expected for hot stripped stars (Wang et al. 2018, 2021). Furthermore, in observations, there may be hints of a slight trend of increasing secondary masses with larger primary masses, a trend which is expected in stripped-star evolutionary models (Shao \& $\mathrm{Li}$ 2021). Finally, the range of observed masses is compatible with that expected for stripped stars (Shao \& Li 2014; Wang et al. 2021).

Secondly, compact objects are also possible in principle, but a single type cannot explain all companions. Indeed, neutron stars tend to have masses of 1-2.5 $M_{\odot}$ (Alsing et al. 2018) and can thus only explain the most massive (and rarer) companions. In contrast, white dwarfs generally have masses $<1 M_{\odot}$ (Nalezyty et al. 2005; Kilic et al. 2018) and, even if a few cases with masses just below Chandrasekhar's limit are known (e.g. Caiazzo et al. 2021), they certainly cannot explain the most massive companions $\left(>1.5 M_{\odot}\right)$.

We are thus left with the stripped stars as the most probable nature for the companions of Be stars, including $\gamma$ Cas stars, if a single type of companions were to explain all binaries. Which impact does this conclusion have on the nature of the $\gamma$ Cas phenomenon? Probably the scenario with a NS in propeller stage can now be discarded, as the low masses of most detected companions of $\gamma$ Cas stars are not compatible with a NS nature (see also Smith et al. 2017, for other arguments). Furthermore, the fact that $\gamma$ Cas stars do not appear significantly different from other Be stars - as much as the small number statistics seems to suggest - may not be favorable to scenarios in which the companion is responsible of the $\gamma$ Cas phenomenon. Indeed, if the vast majority of $\mathrm{Be}$ stars lie in binaries and if the companion is responsible for the $\gamma$ Cas phenomenon, then the vast majority of $\mathrm{Be}$ systems should display the same X-ray characteristics. This is not indicated by X-ray surveys of Be stars (Nazé \& Motch 2018). This is also not suggested by the quite strict upper limits $\log \left(L_{\mathrm{X}} / L_{\mathrm{BOL}}\right)<$ -7.3 (or lower) on X-ray luminosities derived by Berghoefer et al. (1996) for eight stars at the bottom of Table 4. Certainly more research is now needed to understand the $\gamma$ Cas phenomenon. For example, to maintain a binary scenario would require some specific difference for the $\gamma$ Cas systems (not period/separation, eccentricity, or secondary mass - but maybe peculiar disk/orbit configurations or some other particular property?).

A final piece of information is that the peculiar tangential velocities of stars studied in this paper, derived from GAIA-eDR3, are all below $20 \mathrm{~km} \mathrm{~s}^{-1}$. Therefore, none of them seems to be a runaway although Berger \& Gies (2001) had proposed such a status for V782 Cas. 


\subsection{Higher multiplicity}

Spectroscopy and photometry reveal the presence of two periods ( $2.5 \mathrm{~d}$ and $122 \mathrm{~d})$ in V782 Cas data, hinting at a high multiplicity. Our analysis shows that the short-period system is composed of two B-stars but there is no evidence that this binary is orbited on the longer timescale by the Be star, suggesting the system to be quadruple.

High multiplicity may actually be common for massive O-stars (Sana et al. 2014). Moreover, this is not the first time that high multiplicity is reported for Be stars. Several examples of visual companions have been reported, even for $\gamma$ Cas itself $^{7}$. Additional companions have been proposed following spectroscopic analyses. In their study of $o$ Cas (a system with $P \sim 1032$ d), Koubský et al. (2010) concluded that, to explain its too large mass, the companion to the Be primary actually may be a short-period $(P \sim 1.26 \mathrm{~d}) \mathrm{A}+\mathrm{A}$ binary. For CW Cep and $66 \mathrm{Oph}$, periods of $2.7 \mathrm{~d}$ and $10.78 \mathrm{~d}$ were reported by Stickland et al. (1992) and Štefl et al. (2004), respectively. The lack of motion for the $\mathrm{H} \alpha$ line of these two Be stars suggested to Rivinius et al. (2020) and Štefl et al. (2004), respectively, that the Be star was a distant companion to an inner, short-period massive binary composed of two B-type stars (early-type for CW Cep and late-type for $66 \mathrm{Oph}$ ). Note that both systems also have visual companions which are even further away. From a similar observation, Rivinius et al. (2020) arrived at the same conclusion for LB-1 and for HR 6819. However, these results ignited some lively discussions. For example, Johnston et al. (2019) rather interpreted the lack of velocity shifts in $\mathrm{H} \alpha$ as the sign of its arising in a circumbinary envelope: these authors considered CW Cep to be a binary, not a triple. In addition, Shenar et al. (2020) and Gies \& Wang (2020) reported reflex binary motions for $\mathrm{H} \alpha$ in LB-1 and HR 6819, respectively, discarding the "outer Be in a triple system" interpretation in favor of a more classical Be binary scenario.

There are however less disputed cases of triple-star systems involving a Be star. Miroshnichenko et al. (2013) reported on a long-term spectroscopic monitoring of $\delta$ Sco. It comprises a Be star and a B13 companion in a highly eccentric orbit of period of $10.8 \mathrm{yrs}$, but longer-term velocity variations indicate the presence of a third star in the system. In parallel, the spectrum of HD 93683 indicates not only short-term binary motion linked to an inner O9V+B0V binary with $P \sim 18 \mathrm{~d}$, but also long-term velocity shifts in the $\mathrm{H} \alpha$ line, suggesting the presence of an outer Be star with a period of about $400 \mathrm{~d}$ (Bodensteiner et al. 2020). Interferometric and spectroscopic data further indicate that $v$ Gem comprises an inner B $+\mathrm{B}$ binary with period $54 \mathrm{~d}$ orbited in $19 \mathrm{yr}$ by an outer Be star (Gardner et al. 2021; Klement et al. 2021).

The landscape of multiple systems comprising a Be star appears quite varied and, due to small number statistics, it is difficult to assess how similar/different V782 Cas is, compared to previously known cases.

\section{CONCLUSION}

We used high-resolution spectroscopy (TIGRE, CARMENES, UVES) to assess the multiplicity of a large sample of $\gamma$ Cas stars. The remaining objects are either known to be binaries - two cases - or too faint for a thorough study with current facilities. An SB1 orbital solution could be established for six $\gamma$ Cas stars, with one of them actually being a rare candidate of quadruple system. A further

\footnotetext{
7 Mamajek (2017) proposed this star to be triple and associated to the quadruple HD 5408
}

five stars show velocity shifts reminiscent of orbital motion, although more data are needed to fully establish the orbit. Combining these results with previous ones ( $\gamma$ Cas and $\pi$ Aqr being known binaries) implies that at least a half of the studied $\gamma$ Cas stars have companions and a further quarter are promising binary candidates.

The derived parameters are in line with those found in other Be binaries: long periods, small eccentricities, and low-mass companions. This suggests that a single type of compact objects are probably not a good way to explain the $\gamma$ Cas phenomenon as a whole. Also, the particularly bright and hard thermal X-ray emission remains the sole difference between $\gamma$ Cas stars and other Be stars: the simple presence of companions cannot explain this peculiarity.

\section{ACKNOWLEDGEMENTS}

The authors acknowledge interesting discussions with Pr Anatoly Miroshnichenko and thank Pr Andrei Tokovinin for his constructive report. Y.N. and G.R. acknowledge support from the Fonds National de la Recherche Scientifique (Belgium), the European Space Agency (ESA) and the Belgian Federal Science Policy Office (BELSPO) in the framework of the PRODEX Programme (contracts linked to XMM-Newton and Gaia). ADS and CDS were used for preparing this document. TIGRE is a collaboration of the Hamburger Sternwarte, the Universities of Hamburg, Guanajuato and Liège. This project is based on observations collected at CAHA (proposal H193.5-051+21B-3.5-051) operated jointly by junta de Andalucia and Consejo Superiod de Investigationes Cientificas (CSIC). Because it relies on OPTICON transnational access (program ID 19B/001), this project has received funding from the European Union's Horizon 2020 research and innovation programme under grant agreements No $730890+101004719$. This material reflects only the authors views and the Commission is not liable for any use that may be made of the information contained therein.

\section{DATA AVAILABILITY}

The ESO and Calar Alto (CARMENES) data used in this article are available in their respective public archives. The TIGRE data are available upon reasonable request.

\section{REFERENCES}

Abt, H. A. \& Levy, S. G. 1978, ApJS, 36, 241. doi:10.1086/190498

Abt, H. A. \& Cardona, O. 1984, ApJ, 285, 190. doi:10.1086/162490

Aufdenberg, J. P. 1994, AAS, 185, 2110

Alsing, J., Silva, H. O., \& Berti, E. 2018, MNRAS, 478, 1377. doi:10.1093/mnras/sty 1065

Baade, D. 1992, Evolutionary Processes in Interacting Binary Stars, 151, 147

Becker, J. C., Johnson, J. A., Vanderburg, A., et al. 2015, ApJS, 217, 29. doi:10.1088/0067-0049/217/2/29

Berger, D. H. \& Gies, D. R. 2001, ApJ, 555, 364. doi:10.1086/321461

Berghoefer, T. W., Schmitt, J. H. M. M., \& Cassinelli, J. P. 1996, A\&AS, 118, 481

Bjorkman, K. S., Miroshnichenko, A. S., McDavid, D., \& Pogrosheva, T. M. 2002, ApJ, 573, 812

Bodensteiner, J., Shenar, T., \& Sana, H. 2020, A\&A, 641, A42. doi:10.1051/0004-6361/202037640

Boubert, D. \& Evans, N. W. 2018, MNRAS, 477, 5261. doi:10.1093/mnras/sty980

Caballero, J. A., Guàrdia, J., López del Fresno, M., et al. 2016, Proc. SPIE, 9910, 99100E 
Caiazzo, I., Burdge, K. B., Fuller, J., et al. 2021, Nature, 595, 39

Cantiello, M., Langer, N., Brott, I., et al. 2009, A\&A, 499, 279

Carrier, F., Burki, G., \& Burnet, M. 2002, A\&A, 385, 488. doi:10.1051/00046361:20020174

Chojnowski, S. D., Wisniewski, J. P., Whelan, D. G., et al. 2017, AJ, 153, 174. doi:10.3847/1538-3881/aa64ce

Chojnowski, S. D., Labadie-Bartz, J., Rivinius, T., et al. 2018, ApJ, 865, 76. doi:10.3847/1538-4357/aad964

Cincotta, P. M., Helmi, A., Mendez, M., Nunez, J. A., \& Vucetich, H. 1999, MNRAS, 302, 582

Cincotta, P. M. 1999, MNRAS, 307, 941

Cracco, V., Orio, M., Ciroi, S., et al. 2018, ApJ, 862, 167. doi:10.3847/15384357/aacefb

Dekker, H., D’Odorico, S., Kaufer, A., et al. 2000, Proc. SPIE, 534

Dosopoulou, F. \& Kalogera, V. 2016, ApJ, 825, 71. doi:10.3847/0004$637 \mathrm{X} / 825 / 1 / 71$

Drilling, J.S., \& Landolt, A.U. (2000), in Allen's Astrophysical Quantities, 4th edition, A.N. Cox (ed.), 381

Eggleton, P.P. 1983, ApJ, 268, 368

Ekström, S., Meynet, G., Maeder, A., et al. 2008, A\&A, 478, 467. doi:10.1051/0004-6361:20078095

Elias, N. M., Wilson, R. E., Olson, E. C., et al. 1997, ApJ, 484, 394. doi: $10.1086 / 304299$

Frémat, Y., Zorec, J., Hubert, A.-M., et al. 2005, A\&A, 440, 305. doi:10.1051/0004-6361:20042229

Gardner, T., Monnier, J. D., Fekel, F. C., et al. 2021, AJ, 161, 40. doi:10.3847/1538-3881/abcf4e

Gies, D. R. \& Bolton, C. T. 1986, ApJS, 61, 419. doi:10.1086/191118

Gies, D. R. \& Wang, L. 2020, ApJ, 898, L44. doi:10.3847/2041-8213/aba51c

González, J.F., \& Levato, H. 2006, A\&A 448, 283

Graham, M. J., Drake, A. J., Djorgovski, S. G., Mahabal, A. A., \& Donalek, C. 2013, MNRAS, 434, 2629

Gosset, E., Royer, P., Rauw, G., et al. 2001, MNRAS, 327, 435

Hamaguchi, K., Oskinova, L., Russell, C. M. P., et al. 2016, ApJ, 832, 140

Harmanec, P. 1987, Bulletin of the Astronomical Institutes of Czechoslovakia, 38,283

Harmanec, P., Koubský, P., Nemravová, J. A., et al. 2015, A\&A, 573, A107. doi:10.1051/0004-6361/201424640

Hastings, B., Langer, N., Wang, C., et al. 2021, A\&A, in press, arXiv:2106.12263

Heck, A., Manfroid, J., \& Mersch, G. 1985, A\&AS, 59, 63

Hinkle, K., Wallace, L., Valenti, J., \& Harmer, D. 2000, Visible and Near Infrared Atlas of the Arcturus Spectrum 3727-9300 A ed. Kenneth Hinkle, Lloyd Wallace, Jeff Valenti, and Dianne Harmer. (San Francisco: ASP) ISBN: 1-58381-037-4, 2000.,

Horch, E. P., van Belle, G. T., Davidson, J. W., et al. 2020, AJ, 159, 233. doi:10.3847/1538-3881/ab87a6

Johnston, C., Pavlovski, K., \& Tkachenko, A. 2019, A\&A, 628, A25. doi:10.1051/0004-6361/201935235

Kennea, J. A., Coe, M. J., Evans, P. A., et al. 2021, The Astronomer's Telegram, 14341

Kilic, M., Hambly, N. C., Bergeron, P., et al. 2018, MNRAS, 479, L113. doi:10.1093/mnrasl/sly110

Klement, R., Carciofi, A. C., Rivinius, T., et al. 2019, ApJ, 885, 147. doi:10.3847/1538-4357/ab48e7

Klement, R., Hadrava, P., Rivinius, T., et al. 2021, arXiv:2105.13437

Koubský, P., Harmanec, P., Hubert, A. M., et al. 2000, A\&A, 356, 913

Koubský, P., Hummel, C. A., Harmanec, P., et al. 2010, A\&A, 517, A24. doi:10.1051/0004-6361/201014477

Koubský, P., Kotková, L., Votruba, V., et al. 2012, A\&A, 545, A121. doi:10.1051/0004-6361/201219679

Koubský, P., Kotková, L., Kraus, M., et al. 2014, A\&A, 567, A57. doi:10.1051/0004-6361/201424022

Kriz, S. \& Harmanec, P. 1975, Bulletin of the Astronomical Institutes of Czechoslovakia, 26, 65

Labadie-Bartz, J., Pepper, J., McSwain, M. V., et al. 2017, AJ, 153, 252. doi:10.3847/1538-3881/aa6396
Langer, N., Baade, D., Bodensteiner, J., et al. 2020, A\&A, 633, A40. doi:10.1051/0004-6361/201936736

Lanz, T., \& Hubeny, I. 2003, ApJS 147, 225

Lindroos, K. P. 1985, A\&AS, 60, 183

Maíz Apellániz, J. 2010, A\&A, 518, A1. doi:10.1051/0004-6361/201014409

Mamajek, E. 2017, Journal of Double Star Observations, 13, 264

Mason, B. D., Martin, C., Hartkopf, W. I., et al. 1999, AJ, 117, 1890. doi:10.1086/300823

Mason, B. D., Hartkopf, W. I., Gies, D. R., et al. 2009, AJ, 137, 3358. doi:10.1088/0004-6256/137/2/3358

Miroshnichenko, A. S., Pasechnik, A. V., Manset, N., et al. 2013, ApJ, 766, 119. doi:10.1088/0004-637X/766/2/119

Mittag, M., Hempelmann, A., González-Pérez, J.N., \& Schmitt, J.H.M.M. 2010, Adv. Astron. 101502

Motch, C., Lopes de Oliveira, R., \& Smith, M. A. 2015, ApJ, 806, 177

Mourard, D., Monnier, J. D., Meilland, A., et al. 2015, A\&A, 577, A51. doi:10.1051/0004-6361/201425141

Murakami, T., Koyama, K., Inoue, H., \& Agrawal, P. C. 1986, ApJ, 310, L31

Nalezyty, M., Madej, J., \& Althaus, L. G. 2005, 14th European Workshop on White Dwarfs, 334, 107

Nazé, Y., \& Motch, C. 2018, A\&A, 619, A148

Nazé, Y., Rauw, G., \& Smith, M. 2019a, A\&A, 632, A23. doi:10.1051/0004$6361 / 201936307$

Nazé, Y., Rauw, G., Czesla, S., et al. 2019b, A\&A, 627, A99. doi:10.1051/0004-6361/201935141

Nazé, Y., Motch, C., Rauw, G., et al. 2020a, MNRAS, 493, 2511. doi:10.1093/mnras/staa457

Nazé, Y., Pigulski, A., Rauw, G., et al. 2020b, MNRAS, 494, 958. doi:10.1093/mnras/staa617

Nazé, Y., Rauw, G., \& Pigulski, A. 2020c, MNRAS, 498, 3171. doi:10.1093/mnras/staa2553

Nemravová, J., Harmanec, P., Koubský, P., et al. 2012, A\&A, 537, A59

Peters, G. J., Gies, D. R., Grundstrom, E. D., et al. 2008, ApJ, 686, 1280. doi:10.1086/591145

Peters, G. J., Pewett, T. D., Gies, D. R., et al. 2013, ApJ, 765, 2. doi:10.1088/0004-637X/765/1/2

Peters, G. J., Wang, L., Gies, D. R., et al. 2016, ApJ, 828, 47. doi:10.3847/0004-637X/828/1/47

Pols, O. R., Cote, J., Waters, L. B. F. M., et al. 1991, A\&A, 241, 419

Postnov, K., Oskinova, L., \& Torrejón, J. M. 2017, MNRAS, 465, L119

Quirrenbach, A., Amado, P. J., Ribas, I., et al. 2018, Ground-based and Airborne Instrumentation for Astronomy VII, 10702, 107020W

Raguzova, N. V. 2001, A\&A, 367, 848. doi:10.1051/0004-6361:20000348

Rauw, G., Nazé, Y., Smith, M. A., et al. 2018, A\&A, 615, A44

Rivinius, T., Carciofi, A. C., \& Martayan, C. 2013, A\&ARv, 21, 69. doi:10.1007/s00159-013-0069-0

Rivinius, T., Baade, D., Hadrava, P., et al. 2020, A\&A, 637, L3. doi:10.1051/0004-6361/202038020

Roberts, L. C., Turner, N. H., \& ten Brummelaar, T. A. 2007, AJ, 133, 545. doi: $10.1086 / 510335$

Rosu, S., Rauw, G., Conroy, K.E., et al. 2020, A\&A 635, A145

Ruždjak, D., Božić, H., Harmanec, P., et al. 2009, A\&A, 506, 1319. doi:10.1051/0004-6361/200810526

Sana, H., de Mink, S. E., de Koter, A., et al. 2012, Science, 337, 444. doi:10.1126/science.1223344

Sana, H., Le Bouquin, J.-B., Lacour, S., et al. 2014, ApJS, 215, 15. doi:10.1088/0067-0049/215/1/15

Schmitt, J.H.M.M., Schröder, K.-P., Rauw, G., Hempelmann, A., Mittag, M., González-Perez, J.N., \& Czesla, S. 2014, AN, 335, 787

Schwarzenberg-Czerny, A. 1989, MNRAS, 241, 153

Secchi, A. 1866, Astronomische Nachrichten, 68, 63. doi:10.1002/asna.18670680405

Sepinsky, J. F., Willems, B., Kalogera, V., et al. 2009, ApJ, 702, 1387. doi:10.1088/0004-637X/702/2/1387

Shafter, A. W., Szkody, P., \& Thorstensen, J. R. 1986, ApJ, 308, 765

Shao, Y. \& Li, X.-D. 2014, ApJ, 796, 37. doi:10.1088/0004-637X/796/1/37

Shao, Y. \& Li, X.-D. 2021, ApJ, 908, 67. doi:10.3847/1538-4357/abd2b4 
Table A1. List of targets examined in this paper

\begin{tabular}{lccc}
\hline Name & Alt. Name & sp. type & $V$ (mag) \\
\hline V782 Cas & HD 12882 & B2.5III & 7.62 \\
HD 44458 & FR CMa & B1Vpe & 5.55 \\
HD 45995 & & B2Vnne & 6.14 \\
HD 90563 & & B2Ve & 9.86 \\
HD 110432 & BZ Cru & B0.5IVpe & 5.31 \\
HD 119682 & & B0Ve & 7.90 \\
V767 Cen & HD 120991 & B2Ve & 6.10 \\
CQ Cir & HD 130437 & B1Ve & 10.0 \\
HD 157832 & V750 Ara & B2Vne & 6.66 \\
HD 161103 & V3892 Sgr & Oe & 9.13 \\
V771 Sgr & HD 162718 & B3/5ne & 9.16 \\
HD 316568 & & B2IVpe & 9.66 \\
V558 Lyr & HD 183362 & B3Ve & 6.34 \\
SAO 49725 & BD+473129 & B0.5IIIe & 9.27 \\
V2156 Cyg & BD+43 3913 & B1.5Vnne & 8.91 \\
V810 Cas & HD 220058 & B1npe & 8.59 \\
\hline
\end{tabular}

Shenar, T., Bodensteiner, J., Abdul-Masih, M., et al. 2020, A\&A, 639, L6. doi:10.1051/0004-6361/202038275

Smith, M. A., Robinson, R. D., \& Corbet, R. H. D. 1998, ApJ, 503, 877

Smith, M. A., \& Robinson, R. D. 1999, ApJ, 517, 866

Smith, M. A., Lopes de Oliveira, R., Motch, C., et al. 2012, A\&A, 540, A53

Smith, M. A., Lopes de Oliveira, R., \& Motch, C. 2016, Advances in Space Research, 58, 782

Smith, M. A., Lopes de Oliveira, R., \& Motch, C. 2017, MNRAS, 469, 1502. doi:10.1093/mnras/stx926

Stee, P., Meilland, A., Bendjoya, P., et al. 2013, A\&A, 550, A65. doi:10.1051/0004-6361/201220302

Štefl, S., Hadrava, P., Baade, D., et al. 2004, Stellar Rotation, 215, 166

Stickland, D. J., Koch, R. H., \& Pfeiffer, R. J. 1992, The Observatory, 112, 277

van Bever, J. \& Vanbeveren, D. 1997, A\&A, 322, 116

Vieira, R. G., Carciofi, A. C., Bjorkman, J. E., et al. 2017, MNRAS, 464, 3071. doi:10.1093/mnras/stw2542

Walter, R., Lutovinov, A. A., Bozzo, E., et al. 2015, A\&ARv, 23, 2. doi:10.1007/s00159-015-0082-6

Wang, L., Gies, D. R., \& Peters, G. J. 2018, ApJ, 853, 156. doi:10.3847/15384357/aaa4b8

Wang, L., Gies, D. R., Peters, G. J., et al. 2021, AJ, 161, 248. doi:10.3847/1538-3881/abf144

Wichmann, R. 2011, Nightfall: Animated Views of Eclipsing Binary Stars, Astrophysics Source Code Library, record ascl:1106.016

Zechmeister, M. \& Kürster, M. 2009, A\&A, 496, 577

Zorec, J. \& Briot, D. 1997, A\&A, 318, 443

\section{APPENDIX A: SUPPLEMENTARY DATA}

This Appendix provides the list of targets as well as the radial velocities obtained for each star. For V782 Cas B, the velocities correspond to those found from the disentangling. For other targets, the velocities derived by the four methods are listed - note that for correlation, one spectrum per dataset serves as reference and has a zero velocity (there is one dataset per facility). For TIGRE and CARMENES data, the provided velocities were measured on the $\mathrm{H} \alpha$ line while for UVES data, velocities of the $\mathrm{H} \beta$ line are reported as the $\mathrm{H} \alpha$ line may be subject to saturation in some cases. The two exceptions are HD 119682 and V767 Cen for which $\mathrm{H} \beta$ has a complex profile mixing absorption and emission: He I $\lambda 4143 \AA$ velocities are provided instead.
Table A2. Radial velocities measured on TIGRE data for V782 Cas B using the disentangling method (see text for details).

\begin{tabular}{lc}
\hline HJD-2 450 000. & $R V\left(\mathrm{~km} \mathrm{~s}^{-1}\right)$ \\
\hline 8517.575 & 34.03 \\
8660.940 & 21.56 \\
8662.937 & 64.32 \\
8690.964 & 37.86 \\
8709.913 & -48.16 \\
8728.878 & 5.26 \\
8744.749 & -86.37 \\
8760.725 & 29.16 \\
8771.666 & -8.74 \\
8788.816 & 53.08 \\
8806.733 & 13.08 \\
8820.674 & -21.23 \\
8833.632 & 38.96 \\
8848.646 & 30.35 \\
8865.642 & -68.31 \\
8887.597 & -50.39 \\
9023.952 & -65.95 \\
9055.935 & -46.54 \\
9117.801 & 43.62 \\
9142.720 & 25.54 \\
9162.687 & 6.23 \\
9182.662 & -14.19 \\
9203.670 & 38.11 \\
9236.591 & -8.35 \\
9250.620 & -9.60 \\
9418.947 & -12.55 \\
9497.737 & 33.98 \\
\hline &
\end{tabular}

This paper has been typeset from a $\mathrm{T}_{\mathrm{E}} \mathrm{X} / \mathrm{L} \mathrm{T}_{\mathrm{E}} \mathrm{X}$ file prepared by the author. 
Table A3. Radial velocities (in $\mathrm{km} \mathrm{s}^{-1}$ ) measured on the $\mathrm{H} \alpha$ line of V782 Cas A using four methods (first-order moment, mirror, doubleGaussian, correlation - see text for details).

\begin{tabular}{|c|c|c|c|c|}
\hline HJD-2 450000. & $M 1$ & mirror & 2-Gaussian & correlation \\
\hline \multicolumn{5}{|l|}{ TIGRE } \\
\hline 8517.575 & -5.3 & -8.3 & -6.8 & 10.0 \\
\hline 8660.940 & -10.1 & -11.4 & -9.7 & 4.0 \\
\hline 8662.937 & -10.9 & -10.7 & -9.5 & 3.1 \\
\hline 8690.964 & -6.0 & -6.1 & -5.0 & 8.0 \\
\hline 8709.913 & -3.4 & -1.9 & -1.0 & 9.8 \\
\hline 8728.878 & -2.9 & -1.5 & -0.3 & 13.3 \\
\hline 8744.749 & -9.5 & -8.7 & -5.8 & 3.4 \\
\hline 8760.725 & -8.9 & -11.6 & -9.3 & 7.9 \\
\hline 8771.666 & -13.3 & -13.1 & -11.3 & 0.0 \\
\hline 8788.816 & -9.4 & -9.9 & -8.5 & 5.2 \\
\hline 8806.733 & -7.1 & -7.3 & -6.3 & 5.5 \\
\hline 8820.674 & -5.4 & -4.8 & -3.8 & 7.9 \\
\hline 8833.632 & -1.4 & 0.6 & 1.2 & 12.1 \\
\hline 8848.646 & -0.8 & 0.4 & 0.7 & 15.1 \\
\hline 8865.642 & -6.9 & -6.2 & -3.9 & 5.6 \\
\hline 8887.597 & -11.2 & -12.1 & -10.4 & 2.1 \\
\hline 9023.952 & -12.4 & -12.5 & -10.6 & 2.0 \\
\hline 9055.935 & -5.5 & -5.3 & -4.2 & 8.4 \\
\hline 9117.801 & -2.8 & -5.0 & -0.9 & 14.2 \\
\hline 9142.720 & -7.7 & -8.8 & -7.7 & 5.9 \\
\hline 9162.687 & -4.7 & -6.1 & -4.9 & 8.8 \\
\hline 9182.662 & -0.7 & 0.9 & 2.0 & 13.7 \\
\hline 9203.670 & 0.1 & 2.7 & 3.2 & 13.0 \\
\hline 9236.591 & -0.5 & -3.4 & -0.8 & 15.0 \\
\hline 9250.620 & -9.6 & -10.8 & -10.0 & 4.6 \\
\hline 9418.947 & -5.2 & -3.3 & -2.9 & 8.6 \\
\hline 9497.737 & -8.7 & -8.3 & -7.1 & 5.2 \\
\hline \multicolumn{5}{|l|}{ CARMENES } \\
\hline 8696.651 & -7.5 & -6.6 & -5.6 & 0.0 \\
\hline 8712.654 & -2.5 & -0.4 & 0.2 & 3.7 \\
\hline 8733.675 & 0.2 & -0.8 & -0.2 & 9.9 \\
\hline 8756.488 & -4.0 & -7.4 & -4.7 & 4.4 \\
\hline 8775.538 & -11.1 & -11.9 & -10.4 & -5.3 \\
\hline 9416.663 & -3.2 & -2.2 & -2.2 & 4.1 \\
\hline 9457.644 & -1.3 & -2.3 & -1.8 & 7.4 \\
\hline 9477.489 & -0.8 & -2.0 & -0.2 & 7.3 \\
\hline 9502.462 & -6.1 & -6.0 & -5.4 & 0.2 \\
\hline
\end{tabular}

The line was integrated from -550 to $550 \mathrm{~km} \mathrm{~s}^{-1}$ for the moment method. Wings with normalized fluxes between 1.4 and 2.2 were used for the mirror method. The double Gaussians had center velocities $\pm a= \pm 250 \mathrm{~km} \mathrm{~s}^{-1}$.
Table A4. Same as Table A3 but for HD 44458.

\begin{tabular}{lcccc}
\hline HJD-2 450 000. & $M 1$ & mirror & 2-Gaussian & correlation \\
\hline 8447.873 & 13.4 & 13.2 & 13.0 & 0.0 \\
8463.801 & 13.3 & 12.2 & 13.2 & 1.4 \\
8524.664 & 17.0 & 16.8 & 17.0 & 4.4 \\
8548.618 & 17.4 & 19.2 & 19.8 & 6.3 \\
8885.714 & 16.9 & 21.9 & 22.1 & 3.6 \\
8886.624 & 17.9 & 22.1 & 21.6 & 3.2 \\
8899.632 & 15.5 & 19.5 & 18.9 & 1.2 \\
8910.661 & 17.5 & 22.2 & 21.7 & 3.6 \\
8943.622 & 17.1 & 22.3 & 22.0 & 2.5 \\
8946.585 & 15.7 & 21.5 & 21.5 & 1.3 \\
9123.953 & 13.6 & 14.6 & 14.4 & 0.6 \\
9165.846 & 15.2 & 17.7 & 16.8 & 1.2 \\
9195.791 & 13.4 & 17.1 & 17.3 & 0.6 \\
9217.819 & 17.7 & 21.3 & 21.1 & 4.8 \\
9241.688 & 18.1 & 21.7 & 21.4 & 5.2 \\
9247.647 & 17.3 & 21.8 & 21.7 & 5.3 \\
9301.588 & 21.6 & 25.3 & 25.2 & 8.5 \\
9311.583 & 15.5 & 20.6 & 20.9 & 2.3 \\
9494.977 & 19.9 & 20.1 & 20.0 & 7.5 \\
9496.997 & 17.3 & 17.3 & 16.9 & 4.6 \\
\hline
\end{tabular}

The line was integrated from -400 to $400 \mathrm{~km} \mathrm{~s}^{-1}$ for the moment method. Wings with normalized fluxes between 1.6 and 2.8 were used for the mirror method. The double Gaussians had center velocities $\pm a= \pm 200 \mathrm{~km} \mathrm{~s}^{-1}$.

Table A5. Same as Table A3 but for HD 45995.

\begin{tabular}{lcccc}
\hline HJD-2 450 000. & $M 1$ & mirror & 2-Gaussian & correlation \\
\hline 8446.917 & 12.7 & 15.5 & 15.6 & 0.0 \\
8462.819 & 16.7 & 19.2 & 19.1 & 4.8 \\
8520.683 & 22.0 & 25.4 & 24.6 & 9.6 \\
8546.628 & 13.6 & 16.5 & 16.7 & 0.2 \\
8886.720 & 18.6 & 22.3 & 21.0 & 4.4 \\
8898.659 & 25.2 & 30.8 & 30.4 & 12.8 \\
8946.593 & 16.3 & 20.4 & 20.9 & 2.1 \\
8948.628 & 13.7 & 18.4 & 18.3 & -0.7 \\
9112.990 & 24.3 & 30.5 & 30.2 & 11.9 \\
9165.816 & 14.0 & 16.6 & 17.4 & 1.1 \\
9166.823 & 14.7 & 17.1 & 18.0 & 1.8 \\
9195.801 & 20.8 & 23.9 & 23.7 & 9.1 \\
9217.791 & 25.5 & 30.7 & 29.9 & 13.5 \\
9238.713 & 24.7 & 28.6 & 27.6 & 12.3 \\
9248.649 & 18.5 & 21.0 & 20.7 & 5.6 \\
9252.681 & 18.9 & 21.6 & 21.1 & 5.1 \\
9301.606 & 23.1 & 25.5 & 26.1 & 10.8 \\
9312.574 & 26.8 & 31.0 & 31.1 & 14.5 \\
9494.993 & 19.0 & 20.8 & 21.6 & 6.7 \\
\hline
\end{tabular}

The line was integrated from -500 to $500 \mathrm{~km} \mathrm{~s}^{-1}$ for the moment method. Wings with normalized fluxes between 1.4 and 2.2 were used for the mirror method. The double Gaussians had center velocities $\pm a= \pm 220 \mathrm{~km} \mathrm{~s}^{-1}$. 
Table A6. Radial velocities (in $\mathrm{km} \mathrm{s}^{-1}$ ) measured on the $\mathrm{H} \beta$ line of HD 90563 using four methods (first-order moment, mirror, double-Gaussian, correlation - see text for details).

\begin{tabular}{lcccc}
\hline HJD-2 450 000. & $M 1$ & mirror & 2-Gaussian & correlation \\
\hline 9135.849 & -0.6 & 16.3 & 15.5 & 0.0 \\
9150.832 & -1.5 & 11.4 & 13.5 & -1.8 \\
9169.810 & -8.7 & 4.1 & 4.6 & -2.5 \\
9184.718 & -10.5 & 7.1 & 0.6 & 0.0 \\
9200.682 & -6.4 & 12.9 & 2.8 & -5.0 \\
\hline
\end{tabular}

The line was integrated from -400 to $400 \mathrm{~km} \mathrm{~s}^{-1}$ for the moment method. Wings with normalized fluxes between 1.1 and 1.3 were used for the mirror method. The double Gaussians had center velocities $\pm a= \pm 200 \mathrm{~km} \mathrm{~s}^{-1}$.

Table A7. Same as Table A6 but for HD 110432.

\begin{tabular}{lcccc}
\hline HJD-2 450 000. & $M 1$ & mirror & 2-Gaussian & correlation \\
\hline 9174.824 & -2.1 & -5.7 & -5.6 & 0.0 \\
9188.827 & -2.4 & -2.6 & -4.1 & 1.5 \\
9205.744 & -1.2 & -7.3 & -7.8 & 2.9 \\
9225.856 & -4.5 & -17.1 & -14.8 & -2.4 \\
9242.710 & -9.4 & -17.5 & -16.8 & -7.9 \\
\hline
\end{tabular}

The line was integrated from -400 to $400 \mathrm{~km} \mathrm{~s}^{-1}$ for the moment method. Wings with normalized fluxes between 1.2 and 1.6 were used for the mirror method. The double Gaussians had center velocities $\pm a= \pm 200 \mathrm{~km} \mathrm{~s}^{-1}$.

Table A8. Radial velocities (in $\mathrm{km} \mathrm{s}^{-1}$ ) measured on the He r $\lambda 4143 \AA$ line of HD 119682 using four methods (first-order moment, mirror, double-Gaussian, correlation - see text for details).

\begin{tabular}{lcccc}
\hline HJD-2 450 000. & $M 1$ & mirror & 2-Gaussian & correlation \\
\hline 9185.837 & -9.4 & -13.8 & -12.7 & 0.1 \\
9205.842 & -11.4 & -15.3 & -15.4 & -4.4 \\
9242.753 & -12.9 & -18.1 & -15.7 & -5.2 \\
9257.801 & -7.9 & -5.3 & -7.5 & 2.5 \\
9274.662 & -17.1 & -21.4 & -22.1 & -16.3 \\
\hline
\end{tabular}

The line was integrated from -250 to $250 \mathrm{~km} \mathrm{~s}^{-1}$ for the moment method. Wings with normalized fluxes between 0.85 and 0.9 were used for the mirror method. The double Gaussians had center velocities $\pm a= \pm 150 \mathrm{~km} \mathrm{~s}^{-1}$.

Table A9. Same as Table A8 but for V767 Cen.

\begin{tabular}{lcccc}
\hline HJD-2 450 000. & $M 1$ & mirror & 2-Gaussian & correlation \\
\hline 9111.496 & -12.4 & -19.1 & -22.0 & -12.2 \\
9191.842 & -8.0 & -10.0 & -9.3 & -0.5 \\
9206.834 & -7.6 & -14.0 & -14.7 & -4.4 \\
9231.859 & -7.8 & -13.0 & -15.2 & -4.0 \\
9246.777 & -8.0 & -12.1 & -9.6 & 0.0 \\
\hline
\end{tabular}

The line was integrated from -200 to $200 \mathrm{~km} \mathrm{~s}^{-1}$ for the moment method. Wings with normalized fluxes between 0.75 and 0.85 were used for the mirror method. The double Gaussians had center velocities $\pm a= \pm 50 \mathrm{~km} \mathrm{~s}^{-1}$.
Table A10. Same as Table A6 but for CQ Cir.

\begin{tabular}{lcccc}
\hline HJD-2 450 000. & $M 1$ & mirror & 2-Gaussian & correlation \\
\hline 9115.506 & -34.8 & -35.4 & -36.4 & 0.0 \\
9212.831 & -26.8 & -30.1 & -29.2 & 3.1 \\
9240.847 & -44.5 & -40.1 & -43.8 & -5.6 \\
9255.788 & -32.2 & -33.5 & -33.0 & 0.7 \\
9271.693 & -35.5 & -34.7 & -36.2 & -0.2 \\
\hline
\end{tabular}

The line was integrated from -300 to $300 \mathrm{~km} \mathrm{~s}^{-1}$ for the moment method. Wings with normalized fluxes between 1.15 and 1.4 were used for the mirror method. The double Gaussians had center velocities $\pm a= \pm 200 \mathrm{~km} \mathrm{~s}^{-1}$.

Table A11. Same as Table A6 but for HD 157832.

\begin{tabular}{lcccc}
\hline HJD-2 450 000. & $M 1$ & mirror & 2-Gaussian & correlation \\
\hline 9108.595 & 16.4 & 17.8 & 18.1 & 0.0 \\
9123.548 & 16.8 & 15.5 & 16.5 & -0.8 \\
9138.537 & 11.1 & 9.1 & 8.9 & -8.4 \\
9156.510 & 7.0 & 7.7 & 7.5 & -10.3 \\
9246.856 & 7.0 & 7.9 & 7.6 & -11.3 \\
\hline
\end{tabular}

The line was integrated from -250 to $250 \mathrm{~km} \mathrm{~s}^{-1}$ for the moment method. Wings with normalized fluxes between 1.1 and 1.3 were used for the mirror method. The double Gaussians had center velocities $\pm a= \pm 175 \mathrm{~km} \mathrm{~s}^{-1}$.

Table A12. Same as Table A6 but for HD 161103.

\begin{tabular}{lcccc}
\hline HJD-2 450 000. & $M 1$ & mirror & 2-Gaussian & correlation \\
\hline 9108.618 & -12.2 & -12.4 & -1.8 & -4.3 \\
9123.559 & -13.5 & -10.1 & -1.4 & -6.7 \\
9138.546 & -12.3 & -7.7 & -1.4 & -6.7 \\
9257.848 & -7.5 & -7.5 & 4.0 & 2.3 \\
9271.856 & -9.4 & -9.4 & 3.4 & 0.0 \\
\hline
\end{tabular}

The line was integrated from -200 to $200 \mathrm{~km} \mathrm{~s}^{-1}$ for the moment method. Wings with normalized fluxes between 1.3 and 1.6 were used for the mirror method. The double Gaussians had center velocities $\pm a= \pm 150 \mathrm{~km} \mathrm{~s}^{-1}$.

Table A13. Same as Table A6 but for V771 Sgr.

\begin{tabular}{lcccc}
\hline HJD-2 450 000. & $M 1$ & mirror & 2-Gaussian & correlation \\
\hline 9109.590 & -11.6 & -11.9 & -12.0 & 0.0 \\
9123.602 & -13.3 & -14.0 & -13.9 & -2.1 \\
9138.574 & -13.7 & -14.2 & -14.4 & -3.0 \\
9258.870 & -7.8 & -7.4 & -7.4 & 4.2 \\
9282.837 & -6.0 & -5.9 & -5.9 & 6.2 \\
\hline
\end{tabular}

The line was integrated from -250 to $250 \mathrm{~km} \mathrm{~s}^{-1}$ for the moment method. Wings with normalized fluxes between 1.2 and 1.6 were used for the mirror method. The double Gaussians had center velocities $\pm a= \pm 150 \mathrm{~km} \mathrm{~s}^{-1}$. 
Table A14. Same as Table A6 but for HD 316568.

\begin{tabular}{lcccc}
\hline HJD-2 450 000. & $M 1$ & mirror & 2-Gaussian & correlation \\
\hline 9109.607 & -1.9 & -1.6 & -1.6 & 0.0 \\
9124.545 & 7.0 & 7.7 & 7.8 & 9.7 \\
9140.550 & 10.0 & 13.3 & 12.7 & 16.7 \\
9264.834 & 25.3 & 27.4 & 26.7 & 26.9 \\
9291.848 & 18.4 & 22.0 & 22.8 & 24.2 \\
\hline
\end{tabular}

The line was integrated from -200 to $250 \mathrm{~km} \mathrm{~s}^{-1}$ for the moment method. Wings with normalized fluxes between 1.1 and 1.35 were used for the mirror method. The double Gaussians had center velocities $\pm a= \pm 150 \mathrm{~km} \mathrm{~s}^{-1}$.

Table A15. Same as Table A3 but for V558 Lyr.

\begin{tabular}{lcccc}
\hline HJD-2 450 000. & $M 1$ & mirror & 2-Gaussian & correlation \\
\hline 8941.979 & -36.4 & -52.2 & -52.7 & 0.0 \\
8960.952 & -38.1 & -48.7 & -50.1 & 1.6 \\
8982.971 & -30.1 & -41.9 & -42.1 & 11.6 \\
8996.964 & -29.1 & -40.2 & -41.2 & 10.3 \\
9019.846 & -39.8 & -52.3 & -54.1 & 1.2 \\
9024.855 & -38.1 & -55.2 & -56.6 & -1.4 \\
9045.757 & -40.7 & -56.2 & -56.6 & -4.1 \\
9082.668 & -30.2 & -41.6 & -43.3 & 10.0 \\
9116.590 & -39.8 & -55.9 & -57.7 & -4.7 \\
9137.597 & -37.3 & -51.9 & -52.0 & -2.3 \\
9156.558 & -26.1 & -38.0 & -40.1 & 11.3 \\
9175.565 & -30.4 & -42.9 & -44.6 & 7.4 \\
9306.920 & -28.9 & -43.6 & -45.3 & 3.2 \\
9325.934 & -21.1 & -31.0 & -32.2 & 12.6 \\
9436.745 & -19.1 & -28.1 & -27.2 & 12.2 \\
9495.598 & -19.2 & -21.8 & -18.9 & 5.2 \\
\hline
\end{tabular}

The line was integrated from -400 to $400 \mathrm{~km} \mathrm{~s}^{-1}$ for the moment method. Wings with normalized fluxes between 1.5 and 2.5 were used for the mirror method. The double Gaussians had center velocities $\pm a= \pm 240 \mathrm{~km} \mathrm{~s}^{-1}$.

Table A16. Same as Table A3 but for SAO 49725.

\begin{tabular}{lcccc}
\hline HJD-2 450 000. & $M 1$ & mirror & 2-Gaussian & correlation \\
\hline TIGRE & & & & \\
9498.695 & -9.2 & -10.9 & -11.1 & 0.0 \\
\hline CARMENES & & & & \\
8696.596 & -9.5 & -11.4 & -11.4 & 0.0 \\
8712.467 & -11.2 & -13.7 & -13.6 & -3.5 \\
8733.416 & -13.6 & -16.6 & -16.7 & -6.5 \\
8756.447 & -13.0 & -16.3 & -16.3 & -5.9 \\
8775.292 & -10.8 & -13.4 & -13.4 & -4.7 \\
8792.297 & -10.2 & -12.2 & -12.2 & -0.8 \\
9416.612 & -11.0 & -13.5 & -13.5 & -4.6 \\
9441.563 & -12.3 & -15.0 & -15.0 & -4.2 \\
9457.588 & -11.2 & -14.1 & -14.1 & -3.9 \\
9477.442 & -8.9 & -11.1 & -11.1 & -1.9 \\
9502.402 & -8.6 & -10.6 & -10.5 & 1.4 \\
\hline
\end{tabular}

The line was integrated from -300 to $300 \mathrm{~km} \mathrm{~s}^{-1}$ for the moment method. Wings with normalized fluxes between 2.2 and 4.6 were used for the mirror method. The double Gaussians had center velocities $\pm a= \pm 120 \mathrm{~km} \mathrm{~s}^{-1}$.
Table A17. Same as Table A3 but for V2156 Cyg.

\begin{tabular}{lcccc}
\hline HJD-2 450 000. & $M 1$ & mirror & 2-Gaussian & correlation \\
\hline TIGRE & & & & \\
9336.908 & -8.1 & -10.6 & -10.3 & 0.0 \\
9437.820 & -12.9 & -15.3 & -15.4 & -5.0 \\
9497.625 & -10.1 & -12.1 & -12.2 & -1.7 \\
\hline CARMENES & & & & \\
8696.617 & -7.0 & -8.8 & -7.2 & 0.0 \\
8712.486 & -5.2 & -6.0 & -5.1 & 0.7 \\
8756.464 & -11.1 & -14.3 & -13.6 & -4.7 \\
8775.313 & -13.8 & -16.8 & -16.1 & -8.2 \\
8792.342 & -15.8 & -19.0 & -18.2 & -11.0 \\
9416.637 & -17.2 & -19.9 & -20.0 & -13.9 \\
9438.586 & -12.1 & -14.6 & -14.6 & -7.9 \\
9457.614 & -9.2 & -9.4 & -9.0 & -5.7 \\
9477.461 & -6.4 & -6.8 & -6.6 & -2.8 \\
9502.428 & -12.3 & -13.7 & -13.3 & -7.3 \\
\hline The line was integrated from -475 to $475 \mathrm{~km} \mathrm{~s}^{-1}$ for the moment method. Wings with normalized \\
fluxes between 2.2 and 3.6 were used for the mirror method. The double Gaussians had center \\
\multicolumn{5}{r}{ velocities $\pm a= \pm 175 \mathrm{~km} \mathrm{~s}^{-1}$. }
\end{tabular}

Table A18. Same as Table A3 but for V810 Cas.

\begin{tabular}{lcccc}
\hline HJD-2 450 000. & $M 1$ & mirror & 2-Gaussian & correlation \\
\hline TIGRE & & & & \\
9418.885 & -31.8 & -36.8 & -36.1 & 0.0 \\
9446.758 & -20.6 & -23.2 & -23.4 & 15.0 \\
9496.744 & -29.9 & -35.2 & -34.4 & 3.8 \\
\hline CARMENES & & & & \\
8696.635 & -18.3 & -20.9 & -21.2 & 0.0 \\
8712.641 & -21.4 & -24.8 & -24.7 & -2.4 \\
8733.664 & -29.5 & -34.1 & -33.6 & -13.4 \\
8756.478 & -21.7 & -27.5 & -27.6 & -2.2 \\
8775.335 & -18.3 & -20.6 & -20.7 & -0.2 \\
9416.652 & -30.6 & -35.8 & -35.4 & -15.0 \\
9438.557 & -22.3 & -27.5 & -27.3 & -2.2 \\
9457.632 & -18.8 & -21.0 & -21.5 & -0.3 \\
9477.476 & -26.3 & -30.5 & -31.0 & -10.0 \\
9502.448 & -25.6 & -31.8 & -31.8 & -7.3 \\
\hline
\end{tabular}

The line was integrated from -500 to $500 \mathrm{~km} \mathrm{~s}^{-1}$ for the moment method. Wings with normalized fluxes between 1.6 and 2.8 were used for the mirror method. The double Gaussians had center velocities $\pm a= \pm 220 \mathrm{~km} \mathrm{~s}^{-1}$. 